\title{
Differential Galois Approach to the Non-integrability of the Heavy Top Problem
}

\author{
Andrzej J. Maciejewski \\ Institute of Astronomy, University of Zielona Góra, \\ Podgórna 50, PL-65-246 Zielona Góra, Poland, (e-mail: maciejka@astro.ia.uz.zgora.pl) \\ Maria Przybylska \\ INRIA Projet CAFÉ, \\ 2004, Route des Lucioles, B. P. 93, 06902 Sophia Antipolis Cedex, France, \\ and \\ Torun Centre for Astronomy, Nicholaus Copernicus University, \\ Gagarina 11, PL-87-100 Toruń, Poland, (e-mail: Maria.Przybylska@sophia.inria.fr)
}

-To Jean-Pierre Ramis

\begin{abstract}
We study integrability of the Euler-Poisson equations describing the motion of a rigid body with one fixed point in a constant gravity field. Using the Morales-Ramis theory and tools of differential algebra we prove that a symmetric heavy top is integrable only in the classical cases of Euler, Lagrange, and Kovalevskaya and is partially integrable only in the GoryachevChaplygin case. Our proof is alternative to that given by Ziglin (Funktsional. Anal. i Prilozhen., 17(1):8-23, 1983; Funktsional. Anal. i Prilozhen., 31(1):3-11, 95, 1997).
\end{abstract}

\section{Résumé}

Nous étudions l'intégrabilité des équations de Euler-Poisson qui décrivent le mouvement d'un solide rigide avec un point fixe dans un champ gravitationnel constant. En utilisant la théorie de Morales-Ramis et des outils d'algèbre différentielle, nous prouvons qu'un solide symétrique est intégrable seulement dans les cas classiques d'Euler, Lagrange et Kowalevski, et est partiellement intégrable seulement dans le cas Goryatchev-Tchaplygin. Notre preuve est une alternative à celle donnée par Ziglin (Funktsional. Anal. i Prilozhen., 17(1):8-23, 1983; Funktsional. Anal. i Prilozhen., 31(1):3-11, 95, 1997). 


\section{Equations of motion and motivation}

Equations of motion of a rigid body in external fields are usually written in a body fixed frame. Here, we use the following convention. For a vector $v$ we denote by $\mathbf{V}=\left[V_{1}, V_{2}, V_{3}\right]^{T}$ its coordinates in a body fixed frame, and we consider it as a one column matrix. The vector and scalar products of two vectors $v$ and $\boldsymbol{w}$ expressed in terms of the body fixed coordinates are denoted by $[\mathbf{V}, \mathbf{W}]$ and $\langle\mathbf{V}, \mathbf{W}\rangle$, respectively.

We consider a rigid body of mass $m$ located in a constant gravity field of intensity $g$. One point of the body is fixed. The distance between the fixed point and the mass centre of the body is $D$. Assuming that $g D \neq 0$, we choose units in such a way that $\mu:=m g D=1$. The Euler-Poisson equations

$$
\frac{\mathrm{d}}{\mathrm{d} t} \mathbf{M}=[\mathbf{M}, \mathbf{J M}]+[\mathbf{N}, \mathbf{L}], \quad \frac{\mathrm{d}}{\mathrm{d} t} \mathbf{N}=[\mathbf{N}, \mathbf{J M}]
$$

describe the rotational motion of the body. In the above equations $\mathbf{M}$ denotes the angular momentum of the body, $\mathbf{N}$ is the unit in the direction of the gravity field, and $\mathbf{L}$ is the unit vector from the centre of mass of the body to the fixed point; $\mathrm{J}$ is the inverse of the matrix of inertia, so $\Omega:=\mathbf{J M}$ is the angular velocity of the body. The principal moments of inertia $A, B$, and $C$ are eigenvalues of $\mathbf{J}^{-1}$. For our further consideration it is important to notice that in (1) the body fixed frame is unspecified, so we can choose it according to our needs. A body fixed frame in which $\mathbf{J}$ is diagonal is called the principal axes frame. This frame is uniquely defined (up to the numbering of the axes) when $\mathbf{J}$ has no multiple eigenvalues. If $\mathbf{J}$ has a multiple eigenvalue, e.g. when $A=B$, we say that the body is symmetric. Then the principal axes frame is defined up to a rotation around the symmetry axis.

System (1) depends on parameters $A, B, C$, and $\mathbf{L}$ but physical constraints restrict the allowable values of parameters to a set $\mathcal{P} \subset \mathbb{R}^{6}$ defined by the following conditions

$$
\begin{gathered}
A>0, \quad B>0, \quad C>0, \quad\langle\mathbf{L}, \mathbf{L}\rangle=1, \\
A+B \geq C, \quad B+C \geq A, \quad C+A \geq B .
\end{gathered}
$$

Euler-Poisson equations possess three first integrals

$$
\begin{gathered}
H=\frac{1}{2}\langle\mathbf{M}, \mathbf{J} \mathbf{M}\rangle+\langle\mathbf{N}, \mathbf{L}\rangle, \\
H_{1}=\langle\mathbf{M}, \mathbf{N}\rangle, \quad H_{2}=\langle\mathbf{N}, \mathbf{N}\rangle .
\end{gathered}
$$

It is known that on the level

$$
M_{\chi}=\left\{(\mathbf{M}, \mathbf{N}) \in \mathbb{R}^{6} \mid H_{1}=\chi, H_{2}=1\right\}
$$

the Euler-Poisson equations are the Hamiltonian ones, see e.g. [1; 36; 2].

Remark 1. Configuration space of a rigid body with a fixed point is the Lie group $\mathrm{SO}(3, \mathbb{R})$ (all possible orientations of the body with respect to an inertial frame). Thus, classically, the phase space for the problem is $T^{*} \mathrm{SO}(3, \mathbb{R})$. Hence it is a Hamiltonian system with three degrees of freedom and it possesses one additional first integral $\mathrm{H}_{1}$ (first integral $\mathrm{H}_{2}$ is identically equal to one in this formulation). The existence of this 
first integral is related to the symmetry of the problem (rotations around the direction of the gravity field) and this allows to reduce the system by one degree of freedom. The Euler-Poisson equations can be viewed as an effect of reduction of the system on $T^{*} \mathrm{SO}(3, \mathbb{R})$ with respect to this symmetry. The phase space of the reduced problem can be considered as the dual $\mathfrak{g}^{*}$ to Lie algebra $\mathfrak{g}$ of group of rigid motions $G=\mathbb{R}^{3} \rtimes$ $\mathrm{SO}(3, \mathbb{R})$. We can identify $(\mathbf{N}, \mathbf{M})$ as element of $\mathfrak{g}^{*}=\mathbb{R}^{3 *} \rtimes \mathrm{so}(3, \mathbb{R})^{*}$ using standard isomorphisms between so $(3, \mathbb{R})$ and $\mathbb{R}^{3}$, and between $\mathbb{R}^{3 *}$ and $\mathbb{R}^{3}$. Let $(\mathbf{X}, \mathbf{A}) \in G$, with $\mathbf{X} \in \mathbb{R}^{3}$ and $\mathbf{A} \in \mathrm{SO}(3, \mathbb{R})$. Then the coadjoint action $\operatorname{Ad}_{(\mathbf{X}, \mathbf{A})}^{*}: \mathfrak{g}^{*} \rightarrow \mathfrak{g}^{*}$ is defined by

$$
\operatorname{Ad}_{(\mathbf{X}, \mathbf{A})}^{*}(\mathbf{N}, \mathbf{M})=(\mathbf{A N},[\mathbf{X}, \mathbf{A N}]+\mathbf{A M}) .
$$

Functions $H_{1}$ and $H_{2}$ given by (3) are orbits invariant, i.e., on each orbit of coadjoint action

$$
\mathcal{O}_{(\mathbf{N}, \mathbf{M})}:=\bigcup_{(\mathbf{X}, \mathbf{A}) \in G}\{(\mathbf{A N},[\mathbf{X}, \mathbf{A N}]+\mathbf{A M})\}
$$

they have constant values. They are called Casimirs. Orbits of coadjoint action defined above coincide with $M_{\chi}$ which is diffeomorphic to $T \mathbb{S}^{2}$. As it is well known, orbits of a coadjoint action are symplectic manifolds equipped with the standard KostantBerezin-Souriau-Kirillov symplectic structure [18; 44]. On four dimensional orbits $M_{\chi}$ the Euler-Poisson equations are Hamiltonian with $H$ given by (2) as the Hamilton function.

Thus, as a Hamiltonian system on $M_{\chi}$ the Euler-Poisson equations have two degrees of freedom and are integrable on $M_{\chi}$ if there exists an additional first integral $\mathrm{H}_{3}$ which is functionally independent with $H$ on $M_{\chi}$. Equivalently, we say that the EulerPoisson equations are integrable if there exists a first integral $\mathrm{H}_{3}$ which is functionally independent together with $\mathrm{H}, \mathrm{H}_{1}$ and $\mathrm{H}_{2}$.

We say that the Euler-Poisson equations are partially integrable if they are integrable on $M_{0}$.

The known integrable cases are the following:

1. The Euler case (1758) corresponds to the situation when there is no gravity (i.e. when $\mu=0$ ) or $\mathbf{L}=\mathbf{0}$ (the fixed point of the body is the centre of mass). The additional first integral in this case is the total angular momentum $H_{3}=\langle\mathbf{M}, \mathbf{M}\rangle$.

2. In the Lagrange case [26] the body is symmetric (i.e. two of its principal moments of inertial are equal) and the fixed point lies on the symmetry axis. The additional first integral in this case is the projection of the angular momentum onto the symmetry axis. If we assume that $A=B$, then in the Lagrange case $L_{1}=L_{2}=0$, and $H_{3}=M_{3}$.

3. In the Kovalevskaya case [20; 21] the body is symmetric and the principal moment of inertia along the symmetry axis is half of the principal moment of inertia with respect to an axis perpendicular to the symmetry axis. Moreover, the fixed point lies in the principal plane perpendicular to the symmetry axis. If $A=B=2 C$, then (after an appropriate rotation around the symmetry axis) we have in the Kovalevskaya case $L_{2}=L_{3}=0$. The additional first integral has the form

$$
H_{3}=\left(\frac{1}{2}\left(M_{1}^{2}-M_{2}^{2}\right)+N_{1}\right)^{2}+\left(M_{1} M_{2}+N_{2}\right)^{2}
$$


4. In the Goryachev-Chaplygin case [15] the body is symmetric and, as in the Kovalevskaya case, the fixed point lies in the principal plane perpendicular to the symmetry axis. If we assume that the third principal axis is the symmetry axis, then in the Goryachev-Chaplygin case we have $A=B=4 C$ and $L_{2}=L_{3}=0$. In the Goryachev-Chaplygin case equations (1) are integrable only on the level $H_{1}=0$ and the additional first integral has the following form:

$$
H_{3}=M_{3}\left(M_{1}^{2}+M_{2}^{2}\right)+M_{1} N_{3}
$$

For a long time the question if there are other integrable cases of the Euler-Poisson equations except those enumerated above was open, although many leading mathematicians tried to give a positive answer to it.

The problem was completely solved by S.L. Ziglin in a brilliant way. First, in [49] he proved the following.

Theorem 1 (Ziglin, 1980). If $(A-B)(B-C)(C-A) \neq 0$, then the Euler-Poisson system does not admit a real meromorphic first integral which is functionally independent together with $\mathrm{H}, \mathrm{H}_{1}$ and $\mathrm{H}_{2}$.

Later, he developed in [50] an elegant method, which now is called the Ziglin theory, and using it he proved in [51] the following theorem.

Theorem 2 (Ziglin, 1983). The complexified Euler-Poisson system for a symmetric body is integrable on $M_{0}$ with complex meromorphic first integrals only in the four classical cases.

This result was improved in [52].

Theorem 3 (Ziglin, 1997). The Euler-Poisson system for a symmetric body is integrable on $M_{0}$ with real meromorphic first integrals only in the four classical cases.

We know that in the Euler, Lagrange and Kovalevskaya cases the Euler-Poisson system is integrable globally, i.e. the additional first integral exists on an arbitrary symplectic manifold $M_{\chi}$. In the Goryachev-Chaplygin case the Euler-Poisson equations are integrable only on $M_{0}$, and this fact was also proved by Ziglin.

Remark 2. We do not even try to sketch the very rich history of investigations of the problem of the heavy top. We refer here to books $[14 ; 27 ; 23 ;[8]$ and references therein. However, several works where the question of integrability of the problem was investigated are worth mentioning. In [22] it was shown that for a non-symmetric body the Euler-Poisson equations do not admit an additional real analytic first integral which depends analytically on a small parameter $\mu$, see also [23, Ch. III]. For a symmetric body when the ratio of the principal moments is small enough (i.e. for the case of the perturbed spherical pendulum) the non-existence of an additional real analytic first integral was proved [24; 25]. A similar result for a perturbed Lagrange case was shown in $[12$; 6]. A novel, variational approach to proving the non-integrability was elaborated by $S$. V. Bolotin in [7] where he showed the non-existence of an additional real analytic first integral for a symmetric heavy top for the case when the fixed point lies in the equatorial and the ratio of the principal moments of inertia is greater than 4. 
The Ziglin theory is a continuation of the idea of S. N. Kovalevskaya who related the (non)integrability with the behaviour of solutions of the investigated system as functions of the complex time. The main object in the Ziglin theory is the monodromy group of variational equations around a particular non-equilibrium solution. As it was shown by Ziglin, if the investigated system possesses a meromorphic first integral, the monodromy group of variational equations possesses a rational invariant. Thus, for an integrable system, the monodromy group cannot be too 'rich'. The main difficulty in application of the Ziglin theory is connected with the fact that 'except for a few differential equations, e.g., the Riemann equations, Jordan-Pochhammer equations and generalised hypergeometric equations, the monodromy group has not been determined', [45, p. 85]. For other second order differential equations only partial results are known, see e.g. [3; ;9]. Having this in mind, one can notice that the analysis of the variational equations and determination of properties of their monodromy group given by Ziglin in his proof of Theorem 2 is a masterpiece. Later, the Ziglin theory was developed and applied for a study of non-integrability of various systems but, as far as we know, nobody used Ziglin's brilliant technique developed in his proof of Theorem 2.

In the nineties the theory of Ziglin was extended by a differential Galois approach. It was done independently by C. Simó, J. J. Morales-Ruiz, J.-P. Ramis [37; 38; 39; 40] and A. Braider, R. C. Churchill, D. L. Rod and M. F. Singer [10; 4]. Nowadays, this approach is called the Morales-Ramis theory. The key point in this theory is to replace an investigation of the monodromy group of variational equations by a study of their differential Galois group. The main fact from this theory is similar to that of Ziglin: the existence of a meromorphic first integral implies the existence of a rational invariant of the differential Galois group of variational equations. Forgetting about differences in hypotheses in main theorems of both theories, the biggest advantage of the Morales-Ramis theory is connected with the fact that applying it, we have at our disposal developed tools and algorithms of differential algebra. Thanks to this fact, it can be applied more easily.

We applied the Morales-Ramis theory to study integrability of several systems, see e.g. [28; 29; 32; 30; 31; 34; 33], and we notice that obtaining similar results when working only with the monodromy group is questionable, or, at least difficult. This gives us an idea to reanalyse the Ziglin proof of Theorem 2 which is rather long (about 10 pages in [51]). We wanted to present a new, much shorter and simpler proof which is based on the Morales-Ramis theory and tools from differential algebra. In fact, at the beginning, we believed that giving such proof would be a nice and simple exercise but quickly it appeared that we were wrong. A 'naive' application of the Morales-Ramis theory leads quickly to very tedious calculations or unsolvable complications. As our aim was to give an 'elementary' proof, we put a constrain on the arguments which are allowable in it: no computer algebra. Thus we spent a lot of time analysing sources of difficulties and complications, and the aim of this paper is to present our own version of proofs of Theorem 2 and Theorem 3. As we believe, these proofs present the whole power and beauty of the Morales-Ramis theory.

The plan of this paper is following. To make it self-contained in the next section we shortly describe basic facts from the Morales-Ramis and Ziglin theory. We collected more specific results about special linear differential equations in Appendix. In Section [3] we derive the normal variational equation. Sections 4 and [5 contain our proofs of Theorem $[2$ and 3 , In the last section we give several remarks and comments. 


\section{Theory}

Below we only mention basic notions and facts concerning the Ziglin and MoralesRamis theory following [50; 4; 38].

Let us consider a system of differential equations

$$
\frac{\mathrm{d}}{\mathrm{d} t} x=v(x), \quad t \in \mathbb{C}, \quad x \in M,
$$

defined on a complex $n$-dimensional manifold $M$. If $\varphi(t)$ is a non-equilibrium solution of (4), then the maximal analytic continuation of $\varphi(t)$ defines a Riemann surface $\Gamma$ with $t$ as a local coordinate. Together with system (4) we can also consider variational equations (VEs) restricted to $T_{\Gamma} M$, i.e.

$$
\dot{\xi}=T(v) \xi, \quad \xi \in T_{\Gamma} M .
$$

We can always reduce the order of this system by one considering the induced system on the normal bundle $N:=T_{\Gamma} M / T \Gamma$ of $\Gamma$ [50]

$$
\dot{\eta}=\pi_{\star}\left(T(v) \pi^{-1} \eta\right), \quad \eta \in N .
$$

Here $\pi: T_{\Gamma} M \rightarrow N$ is the projection. The system of $s=n-1$ equations obtained in this way yields the so-called normal variational equations (NVEs). The monodromy group $\mathcal{M}$ of system (5) is the image of the fundamental group $\pi_{1}\left(\Gamma, t_{0}\right)$ of $\Gamma$ obtained in the process of continuation of local solutions of (5) defined in a neighbourhood of $t_{0}$ along closed paths with the base point $t_{0}$. By definition, it is obvious that $\mathcal{M} \subset \mathrm{GL}(s, \mathbb{C})$. A non-constant rational function $f(z)$ of $s$ variables $z=\left(z_{1}, \ldots, z_{s}\right)$ is called an integral (or invariant) of the monodromy group if $f(g \cdot z)=f(z)$ for all $g \in \mathcal{M}$.

From the Ziglin theory we need the basic lemma formulated in [50] and then given in an improved form in [52].

Lemma 1. If system (4) possesses a meromorphic first integral defined in a neighbourhood $U \subset M$, such that the fundamental group of $\Gamma$ is generated by loops lying in $U$, then the monodromy group $\mathcal{M}$ of the normal variational equations has a rational first integral.

If system (4) is Hamiltonian, then necessarily $n=2 m$ and $M$ is a symplectic manifold equipped with a symplectic form $\omega$. The right hand sides $v=v_{H}$ of (4) are generated by a single function $H$ called the Hamiltonian of the system. For given $H$ vector field $v_{H}$ is defined by $\omega\left(v_{H}, u\right)=\mathrm{d} H \cdot u$, where $u$ is an arbitrary vector field on $M$. Then, of course, $H$ is a first integral of the system. For a given particular solution $\varphi(t)$ we fix the energy level $E=H(\varphi(t))$. Restricting (4) to this level, we obtain a well defined system on an $(n-1)$ dimensional manifold with a known particular solution $\varphi(t)$. For this restricted system we perform the reduction of order of variational equations. Thus, the normal variational equations for a Hamiltonian system with $m$ degrees of freedom have dimension $s=2(m-1)$ and their monodromy group is a subgroup of $\operatorname{Sp}(s, \mathbb{C})$.

In the Morales-Ramis theory the differential Galois group $\mathcal{G}$ of normal variational equations plays the fundamental role, see [38; 39]. For a precise definition of the differential Galois group and general facts from differential algebra see $[16 ; 41 ; 5$; $35 ; 47]$. 
We can consider $\mathcal{G}$ as a subgroup of $\mathrm{GL}(s, \mathbb{C})$ which acts on fundamental solutions of (5) and does not change polynomial relations among them. In particular, this group maps one fundamental solution to other fundamental solutions. Moreover, it can be shown that $\mathcal{M} \subset \mathcal{G}$ and $\mathcal{G}$ is an algebraic subgroup of $\mathrm{GL}(s, \mathbb{C})$. Thus, it is a union of disjoint connected components. One of them containing the identity is called the identity component of $\mathcal{G}$ and is denoted by $\mathcal{G}^{0}$.

Morales-Ruiz and Ramis formulated a new criterion of non-integrability for Hamiltonian systems in terms of the properties of $\mathcal{G}^{0}$ [38; 39].

Theorem 4. Assume that a Hamiltonian system is meromorphically integrable in the Liouville sense in a neigbourhood of the analytic curve $\Gamma$. Then the identity component of the differential Galois group of NVEs associated with $\Gamma$ is Abelian.

In most applications the Riemann surface $\Gamma$ associated with the particular solution is open. There are many reasons why it is better to work with compact Riemann surfaces. Because of this, it is customary to compactify $\Gamma$ adding to it a finite number of points at infinity. Doing this we need a refined version of Theorem 4 , for details see [38; 39]. However, in the context of this paper, the thesis of the above theorem remains unchanged if instead of $\Gamma$ and the variational equations over $\Gamma$ we work with its compactification.

\section{Particular solutions and variational equations}

To apply the Ziglin or the Morales-Ramis theory we have to know a non-equilibrium solution. Let us assume that the fixed point is located in a principal plane. Then, in fact, we can find a one parameter family of particular solutions which describe a pendulum like motion of the body. For a symmetric body this assumption is not restrictive (if necessary we can rotate the principal axes around the symmetry axis).

We choose the body fixed frame in the following way. Its first two axes lie in the principal plane where the fixed point is located and the first axis has direction from the fixed point to the centre of mass of the body. We call this frame special. A map given by

$$
(\mathbf{M}, \mathbf{N})=(\widetilde{\mathbf{R}}, \mathbf{R} \widetilde{\mathbf{N}}), \quad \mathbf{R} \in \mathrm{SO}(3, \mathbb{R}),
$$

transforms equations (1) to the form

$$
\frac{\mathrm{d}}{\mathrm{d} t} \widetilde{\mathbf{M}}=[\widetilde{\mathbf{M}}, \widetilde{\mathbf{J}} \widetilde{\mathbf{M}}]+[\widetilde{\mathbf{N}}, \widetilde{\mathbf{L}}], \quad \frac{\mathrm{d}}{\mathrm{d} t} \widetilde{\mathbf{N}}=[\widetilde{\mathbf{N}}, \widetilde{\mathbf{J}} \widetilde{\mathbf{M}}],
$$

where

$$
\widetilde{\mathbf{J}}=\mathbf{R}^{T} \mathbf{J} \mathbf{R}, \quad \widetilde{\mathbf{L}}=\mathbf{R}^{T} \mathbf{L} .
$$

Now, if symbols with tilde correspond to the principal axes frame, then, taking into account our assumption about the location of the fixed point, we have

$$
\widetilde{\mathbf{J}}=\left[\begin{array}{ccc}
A^{-1} & 0 & 0 \\
0 & B^{-1} & 0 \\
0 & 0 & C^{-1}
\end{array}\right], \quad \widetilde{\mathbf{L}}=\left[\widetilde{L}_{1}, 0, \widetilde{L}_{3}\right]^{T} .
$$


Taking

$$
\mathbf{R}=\left[\begin{array}{ccc}
\widetilde{L}_{1} & 0 & \widetilde{L}_{3} \\
0 & 1 & 0 \\
-\widetilde{L}_{3} & 0 & \widetilde{L}_{1}
\end{array}\right]
$$

we obtain

$$
\mathbf{L}=[1,0,0]^{T}, \quad \mathbf{J}=\left[\begin{array}{ccc}
a & 0 & 2 d \\
0 & b & 0 \\
2 d & 0 & c
\end{array}\right]
$$

where

$$
\begin{aligned}
a & =\frac{\widetilde{L}_{1}^{2}}{A}+\frac{\widetilde{L}_{3}^{2}}{C}, \quad c=\frac{\widetilde{L}_{1}^{2}}{C}+\frac{\widetilde{L}_{3}^{2}}{A}, \\
2 d & =\left(\frac{1}{C}-\frac{1}{A}\right) \widetilde{L}_{1} \widetilde{L}_{3}, \quad b=\frac{1}{B} .
\end{aligned}
$$

Thus, the prescribed choice of $\mathbf{R}$ corresponds to the transformation from the special to the principal frame. Without loss of generality we can put $b=1$. For a symmetric body we assume that $A=B \neq C$. Under this assumption, if $d=0$, then $\widetilde{L}_{1} \widetilde{L}_{3}=0$, and, in this case, the special frame coincides with the principal frame.

From now on we consider the complexified Euler-Poisson system, i.e. we assume that $(\mathbf{M}, \mathbf{N}) \in \mathbb{C}^{6}$.

\subsection{Case $d \neq 0$}

It is easy to check that manifold

$$
\mathcal{N}:=\left\{(\mathbf{M}, \mathbf{N}) \in \mathbb{C}^{6} \mid M_{1}=M_{3}=N_{2}=0, N_{1}^{2}+N_{3}^{2}=1\right\} \subset M_{0},
$$

is symplectic sub-manifold of $M_{0}$ diffeomorphic to $T \mathbb{S}_{\mathbb{C}}^{1} \subset T \mathbb{S}_{\mathbb{C}}^{2}$ (by $\mathbb{S}_{\mathbb{C}}^{m}$ we denote $m$-dimensional complex sphere). Moreover, $\mathcal{N}$ is invariant with respect to the flow generated by (1). The Euler-Poisson equations restricted to $\mathcal{N}$ have the following form

$$
\frac{\mathrm{d}}{\mathrm{d} t} M_{2}=N_{3}, \quad \frac{\mathrm{d}}{\mathrm{d} t} N_{1}=-M_{2} N_{3}, \quad \frac{\mathrm{d}}{\mathrm{d} t} N_{3}=M_{2} N_{1},
$$

and are Hamiltonian with $H_{\mid \mathcal{N}}$ as the Hamiltonian function. For each level of Hamiltonian $H_{\mid \mathcal{N}}=e:=2 k^{2}-1$, we obtain a phase curve $\Gamma_{k}$. We restrict our attention to curves corresponding to $e \in(-1,1]$ so that $k \in(0,1]$. A solution of system (7) lying on the level $H_{\mid \mathcal{N}}=2 k^{2}-1$ we denote by $\left(M_{2}(t, k), N_{1}(t, k), N_{3}(t, k)\right)$.

For a generic value of $k$ phase curve $\Gamma_{k}$ is an algebraic curve in $\mathbb{C}^{3}\left\{M_{2}, N_{1}, N_{3}\right\}$, and, as intersection of two quadrics

$$
2 k^{2}-1=\frac{1}{2} M_{2}^{2}+N_{1}, \quad N_{1}^{2}+N_{3}^{2}=1,
$$

is an elliptic curve (for $k=1$ it is a rational curve). We can compactify it adding two points at infinity which lie in directions $(0, \pm i, 1)$. Thus, a generic $\Gamma_{k}$ can be considered 
as a torus with two points removed. In our further consideration we work with subfamily $\Gamma_{k}$ with $k \in(0,1)$. Only in Section 5 we refer to the phase curve $\Gamma_{1}$ corresponding to $k=1$.

Equations (7) describe the pendulum-like motions of the body: the symmetry axis of the body remains permanently in one plane and oscillates or rotates in it around the fixed point.

For a point $p=(\mathbf{M}, \mathbf{N}) \in M_{0}$ by $\mathbf{v}=(\mathbf{m}, \mathbf{n})$ we denote a vector in $T_{p} M_{0}$. Variational equations along phase curve $\Gamma_{k}$ have the following form

$$
\frac{\mathrm{d}}{\mathrm{d} t}\left[\begin{array}{c}
m_{1} \\
m_{2} \\
m_{3} \\
n_{1} \\
n_{2} \\
n_{3}
\end{array}\right]=\left[\begin{array}{cccccc}
2 d M_{2} & 0 & (c-1) M_{2} & 0 & 0 & 0 \\
0 & 0 & 0 & 0 & 0 & 1 \\
(1-a) M_{2} & 0 & -2 d M_{2} & 0 & -1 & 0 \\
0 & -N_{3} & 0 & 0 & 0 & -M_{2} \\
a N_{3}-2 d N_{1} & 0 & 2 d N_{3}-c N_{1} & 0 & 0 & 0 \\
0 & N_{1} & 0 & M_{2} & 0 & 0
\end{array}\right]\left[\begin{array}{l}
m_{1} \\
m_{2} \\
m_{3} \\
n_{1} \\
n_{2} \\
n_{3}
\end{array}\right],
$$

where $\left(M_{2}, N_{1}, N_{3}\right)=\left(M_{2}(t, k), N_{1}(t, k), N_{3}(t, k)\right) \in \Gamma_{k}$. They have the following first integrals

$$
h=M_{2} m_{2}+n_{1}, \quad h_{1}=N_{1} m_{1}+N_{3} m_{3}+M_{2} n_{2}, \quad h_{2}=N_{1} n_{1}+N_{3} n_{3} .
$$

As it was shown by Ziglin, the normal variational equations are given by

$$
\begin{aligned}
\frac{\mathrm{d}}{\mathrm{d} t} m_{1} & =2 d M_{2} m_{1}+(c-1) M_{2} m_{3}, \\
\frac{\mathrm{d}}{\mathrm{d} t} m_{3} & =(1-a) M_{2} m_{1}-2 d M_{2} m_{3}-n_{2}, \\
\frac{\mathrm{d}}{\mathrm{d} t} n_{2} & =\left(a N_{3}-2 d N_{1}\right) m_{1}+\left(2 d N_{3}-c N_{1}\right) m_{3}, \\
0 & =N_{1} m_{1}+N_{3} m_{3}+M_{2} n_{2} .
\end{aligned}
$$

We assume that the particular solution is not a stationary point $\left(M_{2}(t, k) \equiv 0, N_{1}(t, k) \equiv\right.$ $\left.\pm 1, N_{3}(t, k) \equiv 0\right)$. Under this assumption we reduce the above system to the form

$$
\begin{aligned}
& \frac{\mathrm{d}}{\mathrm{d} t} m_{1}=2 d M_{2} m_{1}+(c-1) M_{2} m_{3} \\
& \frac{\mathrm{d}}{\mathrm{d} t} m_{3}=\left[\frac{N_{1}}{M_{2}}+(1-a) M_{2}\right] m_{1}+\left[\frac{N_{3}}{M_{2}}-2 d M_{2}\right] m_{3} .
\end{aligned}
$$

We can write the above system as one second order equation

$$
\frac{\mathrm{d}^{2}}{\mathrm{~d} t^{2}} m+a_{1}(t) \frac{\mathrm{d}}{\mathrm{d} t} m+a_{0}(t) m=0, \quad m \equiv m_{1},
$$

with coefficients

$$
a_{1}(t)=-2 \frac{N_{3}(t, k)}{M_{2}(t, k)}, \quad a_{0}(t)=(1-c) N_{1}(t, k)+2 d N_{3}(t, k)+f M_{2}(t, k)^{2},
$$

and

$$
f=(a-1)(c-1)-4 d^{2}
$$


Now, we make the following transformation of independent variable which is rational parametrisation of the complex circle $\mathbb{S}_{\mathbb{C}}^{1}$

$$
t \longrightarrow z:=\frac{N_{3}(t, k)}{1+N_{1}(t, k)} .
$$

Then we obtain

$$
\begin{gathered}
N_{1}=\frac{1-z^{2}}{1+z^{2}}, \quad N_{3}=\frac{2 z}{1+z^{2}}, \quad M_{2}=\frac{2 \dot{z}}{1+z^{2}}, \\
\dot{z}^{2}=\frac{1}{s^{2}+1}\left(z^{2}+1\right)\left(z^{2}-s^{2}\right) .
\end{gathered}
$$

where

$$
s=\sqrt{\frac{1-e}{1+e}}=\frac{k^{\prime}}{k} .
$$

After transformation (10) equation (9) reads

$$
m^{\prime \prime}+p(z) m^{\prime}+q(z) m=0, \quad '=\frac{\mathrm{d}}{\mathrm{d} z},
$$

with coefficients

$$
p(z)=\frac{1}{2}\left[\frac{3}{z-\mathrm{i}}+\frac{3}{z+\mathrm{i}}-\frac{1}{z-s}-\frac{1}{z+s}\right],
$$

and

$$
q(z)=\sum_{i=1}^{4} \frac{\alpha_{i}}{\left(z-z_{i}\right)^{2}}+\frac{\beta_{i}}{z-z_{i}}
$$

where we denote $\left(z_{1}, z_{2}, z_{3}, z_{4}\right)=(\mathrm{i},-\mathrm{i}, \mathrm{s},-\mathrm{s})$, and

$$
\begin{gathered}
\alpha_{1}=\frac{1}{2}(1-c)-f+\mathrm{i} d, \quad \alpha_{2}=\alpha_{1}^{*}, \quad \alpha_{3}=\alpha_{4}=0, \\
\beta_{1}=-\frac{2 d}{1+s^{2}}-\mathrm{i}\left(f+\frac{c-1}{1+s^{2}}\right), \quad \beta_{2}=\beta_{1}^{*}, \\
\beta_{3}=\frac{(1-c)\left(1-s^{2}\right)+4 d s}{2 s\left(1+s^{2}\right)}, \quad \beta_{4}=\frac{(c-1)\left(1-s^{2}\right)+4 d s}{2 s\left(1+s^{2}\right)} .
\end{gathered}
$$

We can see that equation (11) is Fuchsian and it has four regular singular points $z_{i}$ over the Riemann sphere $\mathbb{C P}^{1}$. The infinity is an ordinary point for this equation. We assumed that $k \in(0,1)$, so $s \in(0, \infty)$. Here it is important to notice that for real values of $a, c$, and $d$, and for all $s \in(0, \infty)$ equation (11) has four distinct regular singular points, i.e, the number of singular points does not depend on $s$. For further calculations we fix $s=1$.

Let us note here that transformation (10) is a branched double covering of Riemann sphere $\mathbb{C P}^{1} \rightarrow \Gamma_{k}$. Moreover, the branching points of this covering are precisely the four points where equation (11) has singularities.

Making the following change of the dependent variable

$$
m=w \exp \left[-\frac{1}{2} \int_{z_{0}}^{z} p(\zeta) \mathrm{d} \zeta\right],
$$


we can simplify (11) to the standard reduced form

$$
w^{\prime \prime}=r(z) w, \quad r(z)=\frac{1}{2} p^{\prime}(z)+\frac{1}{4} p(z)^{2}-q(z),
$$

where $r(z)$ can be written as

$$
r(z)=\sum_{i=1}^{4}\left[\frac{a_{i}}{\left(z-z_{i}\right)^{2}}+\frac{b_{i}}{z-z_{i}}\right]
$$

with coefficients

$$
\begin{gathered}
a_{2}=a_{1}^{*}=F-\frac{1}{4}+\mathrm{i} d, \quad a_{3}=a_{4}=\frac{5}{16}, \\
b_{1}=b_{2}^{*}=d+\mathrm{i}\left(F-\frac{1}{4}\right), \\
b_{3,4}=\mp \frac{5}{16}-d, \quad F=f+\frac{c}{2}-\frac{7}{16} .
\end{gathered}
$$

The differences of exponents $\Delta_{i}=\sqrt{1+4 a_{i}}$ at singular point $z_{i}$ are the following

$$
\Delta_{1}=\Delta_{2}^{*}=\sqrt{F-\mathrm{i} d}, \quad \Delta_{3}=\Delta_{4}=\frac{3}{2} .
$$

\subsection{Case $L_{3}=0$}

Let us assume that $L_{3}=0$. Then, obviously $d=0$, and, as we already mentioned, the special frame coincides with the principal axes frame. As we consider a symmetric body with $A=B=1$, then additionally we have $f=0$, and

$$
F=\frac{c}{2}-\frac{7}{16}, \quad c=\frac{1}{C}
$$

Thanks to that equation (12) has a simpler form and it can be transformed to a Riemann $P$ equation. Instead of making a direct transformation in (12) it is more convenient to start from equation (9). Then, instead of transformation (10) we make the following one

$$
t \mapsto z:=N_{1}(t, k)^{2}
$$

and we obtain

$$
\frac{\mathrm{d}^{2}}{\mathrm{~d} t^{2}} m+\frac{1}{2}\left(\frac{1}{z-1}+\frac{1}{2 z}\right) \frac{\mathrm{d}}{\mathrm{d} t} m+\frac{1-c}{8}\left(\frac{1}{z-1}-\frac{1}{z}\right) m=0 .
$$

For this Riemann $P$ equation the difference of exponents at $z=0$ is $3 / 4$, at $z=1$ is $1 / 2$, and at $z=\infty$ is

$$
\Delta_{\infty}=\frac{1}{4} \sqrt{8 c-7}
$$

Let us notice that as in the case $d \neq 0$ transformation (15) is a branched covering of Riemann sphere $\mathbb{C P}^{1} \rightarrow \Gamma_{k}$, and the branching points of this covering are precisely the three points where equation (16) has singularities. 


\subsection{Case $L_{3}=0$. Second particular solution.}

When $L_{3}=0$ we have at our disposal another family of particular solutions. Under our assumption $A=B=1$ and $\mathbf{L}=[1,0,0]^{T}$, the following manifold

$$
\mathcal{N}_{1}:=\left\{(\mathbf{M}, \mathbf{N}) \in \mathbb{C}^{6} \mid M_{1}=M_{2}=N_{3}=0, N_{1}^{2}+N_{2}^{2}=1\right\} \subset M_{0},
$$

is invariant with respect to the flow of system (1). Similarly as $\mathcal{N}$, manifold $\mathcal{N}_{1}$ is diffeomorphic to $T \mathbb{S}_{\mathbb{C}}^{1} \subset T \mathbb{S}_{\mathbb{C}}^{2}$ and it is a symplectic sub-manifold of $M_{0}$. The EulerPoisson equations restricted to $\mathcal{N}_{1}$, read

$$
\frac{\mathrm{d}}{\mathrm{d} t} M_{3}=-N_{2}, \quad \frac{\mathrm{d}}{\mathrm{d} t} N_{1}=c M_{3} N_{2}, \quad \frac{\mathrm{d}}{\mathrm{d} t} N_{2}=-c M_{3} N_{1} .
$$

We consider a family of phase curves $k \mapsto \Gamma_{k}^{1}$ of the above equations given by

$$
\frac{1}{2} c M_{3}^{2}+N_{1}=e, \quad N_{1}^{2}+N_{2}^{2}=1,
$$

where $e=2 k^{2}-1$. For $k \in(0,1)$ curves $\Gamma_{k}^{1}$ are non-degenerate elliptic curves. Variational equations along phase curve $\Gamma_{k}^{1}$ have the following form

$$
\frac{\mathrm{d}}{\mathrm{d} t}\left[\begin{array}{c}
m_{1} \\
m_{2} \\
m_{3} \\
n_{1} \\
n_{2} \\
n_{3}
\end{array}\right]=\left[\begin{array}{cccccc}
0 & (c-1) M_{3} & 0 & 0 & 0 & 0 \\
(1-c) M_{3} & 0 & 0 & 0 & 0 & 1 \\
0 & 0 & 0 & 0 & -1 & 0 \\
0 & 0 & c N_{2} & 0 & c M_{3} & 0 \\
0 & 0 & -c N_{1} & -c M_{3} & 0 & 0 \\
-N_{2} & N_{1} & 0 & 0 & 0 & 0
\end{array}\right]\left[\begin{array}{c}
m_{1} \\
m_{2} \\
m_{3} \\
n_{1} \\
n_{2} \\
n_{3}
\end{array}\right],
$$

and they have the following first integrals

$$
\begin{aligned}
h & =c M_{3} m_{3}+n_{1}, \\
h_{1} & =N_{1} m_{1}+N_{2} m_{2}+M_{3} n_{3}, \\
h_{2} & =N_{1} n_{1}+N_{2} n_{2} .
\end{aligned}
$$

The normal variational equations are given by

$$
\begin{aligned}
\frac{\mathrm{d}}{\mathrm{d} t} m_{1} & =(c-1) M_{3} m_{2}, \\
\frac{\mathrm{d}}{\mathrm{d} t} m_{2} & =(1-c) M_{3} m_{1}+n_{3}, \\
\frac{\mathrm{d}}{\mathrm{d} t} n_{3} & =-N_{2} m_{1}+N_{1} m_{2}, \\
0 & =N_{1} m_{1}+N_{2} m_{2}+M_{3} n_{3} .
\end{aligned}
$$

Now, the reduction of the above system to the second order equation gives

$$
\ddot{n}+\left(M_{3}^{2}-N_{1}\right) n=0, \quad n \equiv n_{3} .
$$

Let us notice that from equations (18) and (19) it follows that

$$
\dot{N}_{1}^{2}=2 c\left(e-N_{1}\right)\left(1-N_{1}^{2}\right) \text {. }
$$


Thus, putting

$$
N_{1}=\frac{2}{c} v+\frac{e}{3}
$$

we obtain the following equation

$$
\dot{v}^{2}=4 v^{3}-g_{2} v-g_{3},
$$

determining the Weierstrass function $v(t)=\wp\left(t ; g_{2}, g_{3}\right)$ with invariants

$$
g_{2}=\frac{1}{3} c^{2}\left(e^{2}+3\right), \quad g_{3}=\frac{1}{27} c^{3} e\left(e^{2}-9\right) .
$$

Hence, we can express $N_{1}$, and $M_{3}^{2}$ (using (19)), in terms of the Weierstrass function $\wp\left(t ; g_{2}, g_{3}\right)$. The discriminant and the modular function of $\wp\left(t ; g_{2}, g_{3}\right)$ are following

$$
\begin{aligned}
& \Delta=g_{2}^{3}-27 g_{3}^{2}=c^{6}\left(e^{2}-1\right)^{2}, \\
& j\left(g_{2}, g_{3}\right)=j(e)=\frac{g_{2}^{3}}{g_{2}^{3}-27 g_{3}^{2}}=\frac{\left(e^{2}+3\right)^{3}}{27\left(e^{2}-1\right)^{2}} .
\end{aligned}
$$

Hence, we can rewrite equation (20) in the form of the Lamé equation

$$
\frac{\mathrm{d}^{2}}{\mathrm{~d} t^{2}} n=\left(\alpha \wp\left(t ; g_{2}, g_{3}\right)+\beta\right) n,
$$

where

$$
\alpha=2 C(2 C+1), \quad \beta=\frac{e}{3} C(1-4 C) .
$$

It is important to notice here the physical restriction on parameter $C$, namely, we have $C \in(0,2)$.

\section{Proof of Theorem 2}

In our proof of Theorem 2 we try to be as close as possible to the proof of Ziglin. Namely, first we show that a necessary condition for integrability is $\widetilde{L}_{3}=0$ (or $\widetilde{L}_{1}=0$, but this gives the already known integrable case of Lagrange). In fact this is the most difficult part of the proof. Then, we use the second family of particular solutions and we restrict the possible values of the principal moment of inertia. Finally, using the first solution, we limit all allowable values of $C$ to those corresponding to the known integrable cases.

We organise the three steps of the proof in the form of three lemmas. Only the first one is somewhat involved, the remaining two are very simple.

The first step is to show that a necessary condition for integrability is $d=0$, see formulae (6).

Lemma 2. Let us assume that $d \neq 0$. Then the identity component of the differential Galois group of equation (12) is not Abelian. 
Proof. In our proof we use of Lemma 8 and 9 , see Appendix. If (12) is reducible then the identity component $\mathcal{G}^{0}$ of its differential Galois group is Abelian in two cases: when $\mathcal{G}$ is a subgroup of diagonal group $\mathcal{D}$ or when it is a proper subgroup of triangular group $\mathcal{T}$.

First we show that $\mathcal{G} \not \subset \mathcal{D}$. Let us assume the opposite. Then there exist two exponential solutions of (12) which have the following form

$$
w_{l}=P_{l} \prod_{i=1}^{4}\left(z-z_{i}\right)^{e_{i, l},} \quad P_{l} \in \mathbb{C}[z], \quad l=1,2,
$$

where $e_{i, l}$ for $l=1,2$ are exponents at singular point $z_{i}$, i.e.,

$$
e_{i, l} \in\left\{\frac{1}{2}\left(1+\Delta_{i}\right), \frac{1}{2}\left(1-\Delta_{i}\right)\right\} .
$$

Here $\Delta_{i}$ for $i=1, \ldots, 4$ are given by (14). The product of these solutions $v=w_{1} w_{2}$ belongs to $\mathbb{C}(z)$ and it is a solution of the second symmetric power of (12), i.e. equation (33) with $r$ given by (13). This equation has the same singular points as equation (12). Exponents $\rho_{i, l}$ at singular points $z_{i}$, and at infinity $\rho_{\infty, l}$ for the second symmetric power of (12) are given by

$$
\rho_{i, l} \in\left\{1,1 \pm \Delta_{i}\right\}, \quad \rho_{\infty, l} \in\{-2,-1,0\}, \quad l=1,2,3,
$$

where $\Delta_{i}$ for $i=1,2,3,4$ are given by (14). If we write $v=P / Q$ with $P, Q \in \mathbb{C}[z]$ then

$$
Q=\prod_{i=1}^{K}\left(z-r_{i}\right)^{n_{i}}, \quad n_{i} \in \mathbb{N}, \quad r_{i} \in\left\{z_{1}, z_{2}, z_{3}, z_{4}\right\},
$$

and $n_{i}=-\rho_{i, l} \in \mathbb{N}$ for certain $l$. However, if $d \neq 0$, then $\rho_{i, l}$ is not a negative integer for $i=1,2,3,4$ and $l=1,2,3$. This implies that $Q=1$. Hence, equation (33) has a polynomial solution $v=P$, and $\operatorname{deg} P=-\rho_{\infty, l} \leq 2$. But $v$ is a product of two exponential solutions of the form (4), so we also have

$$
v=P_{1} P_{2} \prod_{i=1}^{4}\left(z-z_{i}\right)^{e_{i, m}+e_{i, l}} \in \mathbb{C}[z], \quad m, l \in\{1,2\} .
$$

Consequently, $e_{i, m}+e_{i, l}$ is a non-negative integer for $i=1,2,3,4$. As for $d \neq 0$, we have $2 e_{i, l} \notin \mathbb{Z}$ for $i=1,2,3,4$ and $l=1,2$, we deduce that in (22) $m \neq l$. But $e_{i, 1}+e_{i, 2}=1$, for $i=1,2,3,4$. Thus, we have

$$
\operatorname{deg} v=\operatorname{deg} P=\operatorname{deg}\left(P_{1} P_{2}\right)+4 \geq 4 .
$$

We have a contradiction because we already showed that $\operatorname{deg} P \leq 2$.

It is also impossible that $\mathcal{G}$ conjugates to $\mathcal{T}_{m}$ for a certain $m \in \mathbb{N}$ because when $d \neq 0$ exponents for $z_{1}$ and $z_{2}$ are not rational. This implies also that $\mathcal{G}$ is not finite.

The last possibility that $\mathcal{G}^{0}$ is Abelian occurs when $\mathcal{G}$ is conjugated with a subgroup of $D^{\dagger}$. We show that it is impossible. To this end, we apply the second case of the Kovacic algorithm, see Appendix. The auxiliary sets for singular points are following

$$
E_{1}=E_{2}=\{2\}, \quad E_{3}=E_{4}=\{-1,2,5\}, \quad E_{\infty}=\{0,2,4\} .
$$


In the Cartesian product $E=E_{\infty} \times E_{1} \times \cdots \times E_{4}$ we look for such elements $e$ for which

$$
d(e):=\frac{1}{2}\left(e_{\infty}-\sum_{i=1}^{4} e_{i}\right)
$$

is a non-negative integer. There are two such elements, namely

$$
e^{(1)}=(4,2,2,-1,-1), \quad e^{(2)}=(2,2,2,-1,-1) .
$$

We have $d\left(e^{(1)}\right)=1$ and $d\left(e^{(2)}\right)=0$. We have to check if there exists polynomial $P=p_{1} z+p_{0}$ which satisfies the following equation

$$
P^{\prime \prime \prime}+3 \theta P^{\prime \prime}+\left(3 \theta^{2}+3 \theta^{\prime}-4 r\right) P^{\prime}+\left(\theta^{\prime \prime}+3 \theta \theta^{\prime}+\theta^{3}-4 r \theta-2 r^{\prime}\right) P=0,
$$

where

$$
\theta=\frac{1}{z-\mathrm{i}}+\frac{1}{z+\mathrm{i}}-\frac{1}{2} \frac{1}{z-1}-\frac{1}{2} \frac{1}{z+1} .
$$

Inserting $P$ into the above equation we obtain the following system of linear equations for its coefficients

$$
\begin{array}{lr}
-d p_{0}+F p_{1}=0, & (1-2 F) p_{0}+6 d p_{1}=0 \\
F p_{0}+d p_{1}=0, & -6 d p_{0}+(1-2 F) p_{1}=0 .
\end{array}
$$

The above system for $p_{0}$ and $p_{1}$ has a non-zero solution if $d^{2}+F^{2}=0$, but for a real $d$ and $F$ it is possible only when $d=0$ and $F=0$.

As the covering $t \mapsto z$ given by (10) does not change the identity component of the differential Galois group of the normal variational equations (8), from the above lemma it follows that if the Euler-Poisson equations are integrable, then

$$
2 d=\left(\frac{1}{C}-\frac{1}{A}\right) \widetilde{L}_{1} \widetilde{L}_{3}=0 .
$$

For a symmetric body when $A=B \neq C$, the above condition implies that either $\widetilde{L}_{1}=0$, and this corresponds to the integrable case of Lagrange, or $\widetilde{L}_{3}=0$. Hence, we have to investigate the last case. Notice that in this case the special frame is the principal axes frame so we have $\widetilde{\mathbf{L}}=\mathbf{L}=[1,0,0]^{T}, a=1 / A=1 / B=b=1$ and $c=1 / C \neq 1$. At this point it is worth to observe that now the identity component of the differential Galois group of equation (12) is Abelian for infinitely many values of $c$. Let us remind here that from the physical restriction it follows only that $c \in(1 / 2, \infty)$.

Proposition 1. If $L_{3}=0$ then the identity component of the differential Galois group of equation (12) is Abelian in the following cases:

$$
\begin{gathered}
c=1+2 l(4 l-1), \quad c=4+2 l(4 l+5), \quad c=2+2 l(4 l+3,) \\
c=\frac{11}{8}+2 l(l+1), \quad c=\frac{79}{72}+\frac{4}{3} l+2 l^{2},
\end{gathered}
$$

where $l$ is an integer. 
Proof. When $L_{3}=0$ then the identity component of the differential Galois group of equation (12) is Abelian if and only if the differential Galois group of equation (16) is Abelian (transformation from (12) to (16) is algebraic). Then applying Kimura Theorem 5 to equation (16) we easily derive the above values of $c$ for which the identity component of the differential Galois group of this equation is Abelian.

Thus, applying the Morales-Ramis or Ziglin theory and using the first particular solution we cannot prove non-integrability of the Euler-Poison equations for all values of $c$ listed in the above proposition. This is why, in the lemma below, we consider normal variational equations corresponding to the second particular solution.

Lemma 3. Assume that $C \in(0,2)$ and $C \neq m / 4$, for $m=1, \ldots, 7$, then for almost all $e \in \mathbb{R}$, the differential Galois group of equation (21) is $\operatorname{SL}(2, \mathbb{C})$.

Proof. We assume first that $e \neq \pm 1$. Then the discriminant of the elliptic curve associated with $\wp\left(t ; g_{2}, g_{3}\right)$ does not vanish, and we can apply Lemma10, see Appendix. We consider successively three cases from this lemma.

For the Lamé-Hermite case, we have $\alpha=n(n+1)$ for $n \in \mathbb{Z}$. This implies that $C=n / 2$, and hence, as $C \in(0,2)$ we have $C \in\{1 / 2,1,3 / 2\}$.

For the Brioschi-Halphen-Crawford case, we have $\alpha=n(n+1)$ and $m=n+1 / 2 \in$ $\mathbb{N}$. Thus, we have

$$
C=-\frac{1}{4}+\frac{1}{2} m, \quad m \in \mathbb{N} .
$$

So, this case can occur only when $C \in\{1 / 4,3 / 4,5 / 4,7 / 4\}$.

In the Baldassarri case we notice that the mapping

$$
\mathbb{R} \backslash\{-1,1\} \ni e \mapsto j(e),
$$

is non-constant and continuous. Hence, by Dwork Proposition 2 , for a fixed $C$ this case can occur only for a finite number of values of $e$.

As $C=1 / 2, C=1$ and $C=1 / 4$ correspond to the Kovalevskaya, Euler and Goryachev-Chaplygin cases, respectively, we have to investigate cases

$$
C \in\{3 / 4,5 / 4,3 / 2,7 / 4\} .
$$

To this end we return to equation (12). As we show, for $d=0$ it can be transformed to the form (16) and, moreover, this transformation does not change the identity component of its differential Galois group. We can prove the following.

Lemma 4. For $C \in\{3 / 4,5 / 4,3 / 2,7 / 4\}$ the differential Galois group of $(16)$ is $\operatorname{SL}(2, \mathbb{C})$.

Proof. For $C \in\{3 / 4,5 / 4,3 / 2,7 / 4\}$ the respective values of the difference of exponents at infinity $\Delta_{\infty}$ (see formula (17)) for equation (16) are following

$$
\frac{1}{4}\left\{\sqrt{\frac{11}{3}}, \mathrm{i} \sqrt{\frac{3}{5}}, \mathrm{i} \sqrt{\frac{5}{3}}, \mathrm{i} \sqrt{\frac{17}{7}}\right\} .
$$

Now, a direct inspection of possibilities in the Kimura Theorem [5 shows that Riemann $P$ equation (16) with prescribed differences of exponents does not possess a Liouvillian solution, so its differential Galois group is $\operatorname{SL}(2, \mathbb{C})$.

Now the proof of Theorem (2) is a simple consequence of the above three lemmas. 


\section{Proof of Theorem 3}

In the proof of Theorem 3 we apply the Ziglin Lemma 1 and his idea of its application given in [52].

The Euler-Poisson equations restricted to $\mathcal{N}$ possess a hyperbolic equilibrium at $u=(0,0,0,1,0,0)$. The phase curve $\Gamma_{1}$ corresponds to the solution of equations (7) with $k=1$. It contains two real components which are real phase curves corresponding to real solutions homoclinic to $u$. Their union is $\operatorname{Re} \Gamma_{1}$ and we denote its closure by $\Omega$.

Lemma 5. For an arbitrary complex neighbourhood $U \subset \mathcal{N}$ of $\Omega$ there exists $\epsilon>0$, such that for $0<1-k<\epsilon$ the fundamental group $\pi_{1}\left(\Gamma_{k}\right)$ of phase curve $\Gamma_{k}$ is generated by loops lying in $U$.

Proof. The time parametrisation of $\Gamma_{k}$ is given by

$$
\begin{aligned}
M_{2}(t, k) & :=-2 k \operatorname{cn}(t, k), \\
N_{1}(t, k) & :=2 k^{2} \operatorname{sn}^{2}(t, k)-1, \\
N_{3}(t, k) & :=2 k \operatorname{sn}(t, k) \operatorname{dn}(t, k),
\end{aligned}
$$

where $\operatorname{sn}(t, k), \operatorname{cn}(t, k)$ and $\mathrm{dn}(t, k)$ denote the Jacobi elliptic functions of argument $t$ and modulus $k$. Thus, particular solutions of (1)

$$
\varphi(t, k):=\left(0, M_{2}(t, k), 0, N_{1}(t, k), 0, N_{3}(t, k)\right),
$$

defined by (25) are single-valued, meromorphic, and double periodic with periods

$$
T_{1}(k)=2 K(k)+2 \mathrm{i} K^{\prime}(k), \quad T_{2}(k)=2 K(k)-2 \mathrm{i} K^{\prime}(k),
$$

where $K(k)$ is the complete elliptic integral of the first kind with modulus $k, K^{\prime}(k):=$ $K\left(k^{\prime}\right)$, and $k^{\prime}:=\sqrt{1-k^{2}}$. In each period cell they have two simple poles at:

$$
t_{1}(k)=\mathrm{i} K^{\prime}(k), \quad t_{2}(k)=-\mathrm{i} K^{\prime}(k) \bmod \left(T_{1}(k), T_{2}(k)\right) .
$$

Periods $T_{1}(k)$ and $T_{2}(k)$ given by (27) of solution (26) are primitive. Minimal real and imaginary periods are $T(k)=4 K(k)$ and $T^{\prime}(k)=4 \mathrm{i} K^{\prime}(k)$. As a base point $x(k) \in \Gamma_{k}$ we choose $x(k)=\varphi\left(t_{0}(k), k\right)$ where $t_{0}(k)=K(k)$. Let us notice that from (25) it follows that

$$
\begin{aligned}
& M_{2}\left(t_{0}(k), k\right)=0, \\
& N_{1}\left(t_{0}(k), k\right)=2 k^{2}-1, \\
& N_{3}\left(t_{0}(k), k\right)=2 k k^{\prime} .
\end{aligned}
$$

Now, let

$$
\lambda_{k}, \lambda_{k}^{\prime}:[0,1] \rightarrow \Gamma_{k}
$$

be the loops with base point $x(k)$ corresponding to periods $T(k)$ and $T^{\prime}(k)$, respectively. These loops cross at point

$$
x^{\prime}(k)=\varphi\left(t_{0}(k)+T(k) / 2, k\right)=\varphi\left(t_{0}(k)+T^{\prime}(k) / 2, k\right) .
$$




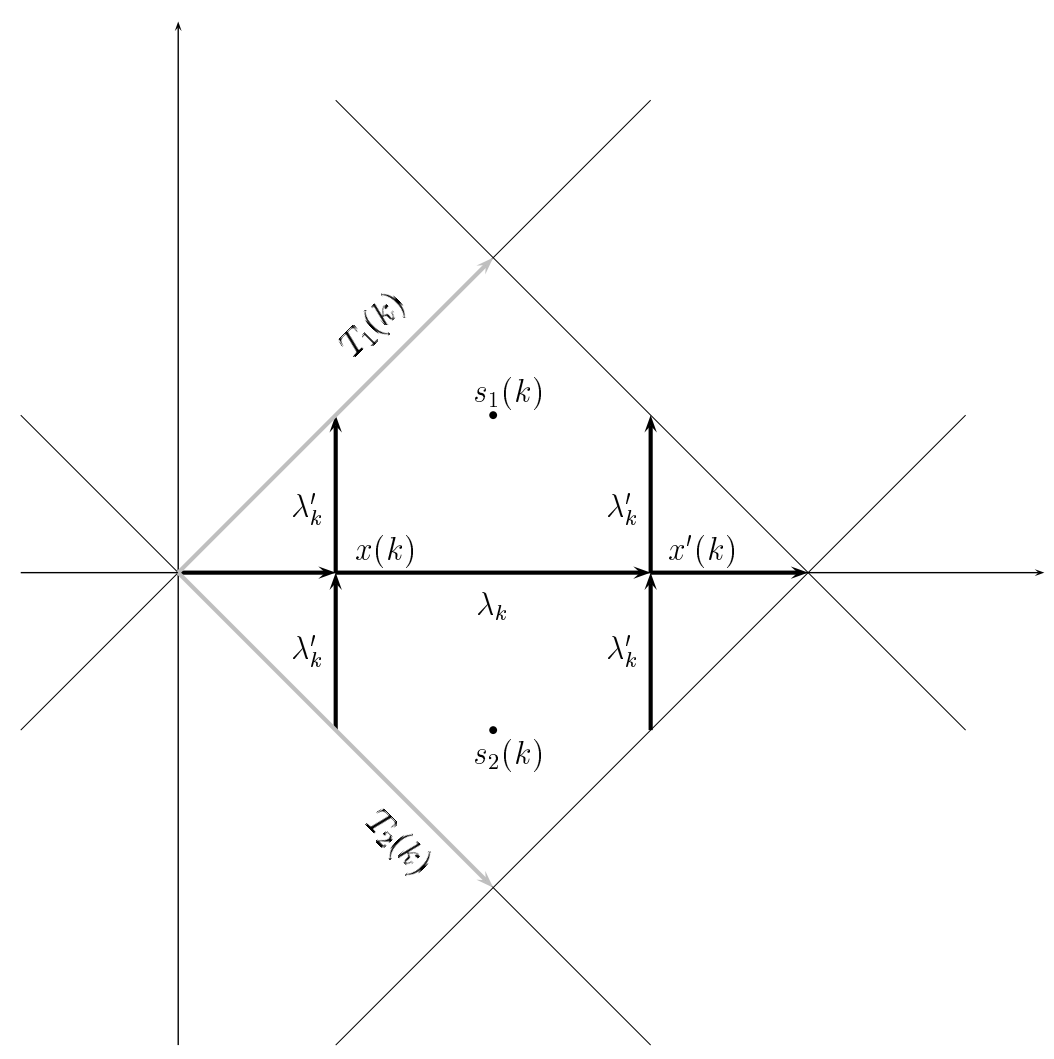

Figure 1: Parallelogram of period with marked loops

As a results, we obtain four semi-loops with end points $x(k)$ and $x^{\prime}(k)$. The fundamental group $\pi_{1}\left(\Gamma_{k}, x(k)\right)$ of $\Gamma_{k}$ is generated by these semi-loops, see Figure 1. Let us analyse what happens when $k$ tends to 1 . From (28) it follows that $x(k)$ tends to $u$ and from (25) we deduce that loop $\lambda_{k}$ tends to $\Omega$. To see what happens with loop $\lambda_{k}^{\prime}$ when $k$ tends to 1 , let us put $t=t_{0}(k)+\mathrm{i} \tau$ in formulae (25). We obtain

$$
\begin{aligned}
& M_{2}(t, k)=2 \mathrm{i} k k^{\prime} \frac{\operatorname{sn}\left(\tau, k^{\prime}\right)}{\operatorname{dn}\left(\tau, k^{\prime}\right)} \\
& N_{1}(t, k)=-1+\frac{2 k^{2}}{\operatorname{dn}^{2}\left(\tau, k^{\prime}\right)} \\
& N_{3}(t, k)=2 k k^{\prime} \frac{\mathrm{cn}^{\prime}\left(\tau, k^{\prime}\right)}{\operatorname{dn}^{2}\left(\tau, k^{\prime}\right)} .
\end{aligned}
$$

Thus, loop $\lambda_{k}^{\prime}$ tends to point $u$ as $k$ tends to 1 .

Time parametrisation of these phase curves $\Gamma_{k}^{1}$ is given by

$$
\begin{aligned}
M_{3}(t, k) & =\frac{2 k}{\omega} \mathrm{cn}(\omega t, k), \\
N_{1}(t, k) & =2 k^{2} \operatorname{sn}^{2}(\omega t, k)-1, \\
N_{2}(t, k) & =2 k \operatorname{sn}(\omega t, k) \operatorname{dn}(\omega t, k),
\end{aligned}
$$

where $\omega^{2}=c$. Thus, the second family of particular solutions of (1)

$$
\varphi_{1}(t, k):=\left(0,0, M_{3}(t, k), N_{1}(t, k), N_{2}(t, k), 0\right),
$$


defined by (29) contains solutions which are single-valued, meromorphic, and double periodic with periods

$$
T_{1}(k)=2 \frac{K(k)}{\omega}+2 \mathrm{i} \frac{K^{\prime}(k)}{\omega}, \quad T_{2}(k)=2 \frac{K(k)}{\omega}-2 \mathrm{i} \frac{K^{\prime}(k)}{\omega} .
$$

In each period cell they have two simple poles at:

$$
t_{1}(k)=\mathrm{i} \frac{K^{\prime}(k)}{\omega}, \quad t_{2}(k)=-\mathrm{i} \frac{K^{\prime}(k)}{\omega} \bmod \left(T_{1}(k), T_{2}(k)\right) .
$$

Let us notice that for the Euler-Poisson equations restricted to $\mathcal{N}_{1}$ point $u=(0,0,0,1,0,0)$ is also a hyperbolic equilibrium. The phase curve $\Gamma_{1}^{1}$ corresponds to the solution of equations (18) with $k=1$. As in the previous case, it contains two real components which are real phase curves corresponding to real solutions homoclinic to $u$. Their union is $\operatorname{Re} \Gamma_{1}^{1}$ and we denote its closure by $\Omega_{1}$. Using the same arguments as in the proof of Lemma 5 we can show the following.

Lemma 6. For an arbitrary complex neighbourhood $U \subset \mathcal{N}_{1}$ of $\Omega_{1}$ there exists $\epsilon>0$, such that for $0<1-k<\epsilon$ the fundamental group $\pi_{1}\left(\Gamma_{k}^{1}\right)$ of phase curve $\Gamma_{k}$ is generated by loops lying in $U$.

Now, to prove Theorem 3 let us notice that we showed that, except for the known integrable cases, the identity component of the differential Galois group of the normal variational equations corresponding to $\Gamma_{k}$ or $\Gamma_{k}^{1}$ is not Abelian for almost all values of $k \in(0,1)$. In fact, in Lemma 2 we proved that for $d \neq 0$ the identity component of the differential Galois group of the normal variation equations corresponding to $\Gamma_{k}$ with $k=1 / \sqrt{2}$ is not Abelian. By Lemma 7 it is not Abelian for almost all values of $k \in(0,1)$. Then, in Lemma 4 we proved that the identity component of the differential Galois group of the normal variation equations corresponding to $\Gamma_{k}^{1}$ is not Abelian for almost all values of $k \in(0,1)$, except for $C=m / 4, m=1, \ldots, 7$. Finally, for $C=m / 4$ such that $C \notin\{1 / 4,1 / 2,1\}$, we showed that the identity component of the differential Galois group of the normal variation equations corresponding to $\Gamma_{k}$ with $k=1 / \sqrt{2}$ is not Abelian. Again, by LemmaZit is not Abelian for almost all values of $k \in(0,1)$.

Both normal variational equations corresponding to $\Gamma_{k}$ and $\Gamma_{k}^{1}$ are Fuchsian. For a Fuchsian equation we know that if the identity component of its differential Galois group is not Abelian then its monodromy group does not possess a rational invariant, see Theorem 3.17 in [4].

Assume now that for $C \notin\{1 / 4,1 / 2,1\}$ the Euler-Poisson equations possess an additional real meromorphic first integral defined in a real neighbourhood of $\Omega \cup \Omega_{1}$. Then we can extend this integral to a complex meromorphic one, defined in a certain complex neighbourhood $U$ of $\Omega \cup \Omega_{1}$. Then, by Lemma 5 and 6 , we find such $\epsilon>0$ that the fundamental groups of $\Gamma_{k}$ and $\Gamma_{k}^{1}$ with $0<1-k<\epsilon$, are generated by loops lying entirely in $U$. Then, from the Ziglin Lemma 1, it follows that both monodromy groups of normal variational equations corresponding to $\Gamma_{k}$ and $\Gamma_{k}^{1}$ possess a rational invariant. However, above we showed that at least for one of them it is not true. A contradiction proves Theorem 3 . 


\section{Remarks and Comments}

One important difference between the Ziglin and Morales-Ramis theory is related with the procedure of obtaining the normal variational equations. Assume that system (4) possesses certain number of known first integrals $H_{i}$, such that their differentials $\mathrm{d} H_{i}$, are linearly independent on $\Gamma$. Then $\mathrm{d}_{i} \circ \pi^{-1}$ for $i=1, \ldots, k$ are independent first integrals of (5). Their common level

$$
N_{p}:=\left\{\eta \in F \mid \mathrm{d} H_{i} \circ \pi^{-1} \eta=p_{i}, \quad p_{i} \in \mathbb{C}, \quad i=1, \ldots, k\right\},
$$

defines a $m$-dimensional linear bundle over $\Gamma$, where $m=n-k-1$. Using these integrals we can reduce the order of system (5). Namely, we consider the reduced normal variational equations

$$
\dot{\eta}=\pi_{\star}\left(T(v) \pi^{-1} \eta\right), \quad \eta \in N_{p}
$$

However after this reduction defined by Ziglin, instead of a linear, we have an affine bundle over $\Gamma$, equations (30) are generally not homogeneous ones, and the monodromy group is a subgroup of affine transformations of $\mathbb{C}^{m}$. Till now this construction has not been translated to the Morales-Ramis theory where we work with a system of homogeneous equations defined on $N_{0}$. To realise the importance of Ziglin reduction let us notice that using only the reduced equation on $N_{0}$ it is impossible to prove global non-integrability of the Goryachev-Chaplygin case. In [52] he gave such a nonintegrability proof investigating the reduced normal variational equations on $N_{p}$ with $p \neq 0$.

The Morales-Ramis theory is coordinate independent, however, investigating a specific problem, we always have to choose appropriate coordinates. The form of normal variational equations depends on local coordinates and this is why their choice is important. It is especially evident when we investigate a problem connected with a rigid body. Equations of motion of the heavy top can be written in many different forms. As we mentioned in Remark 1, the natural phase space for a rigid body with a fixed point is $T^{*} \mathrm{SO}(3, \mathbb{R})$. There are no 'natural' coordinates on $\mathrm{SO}(3, \mathbb{R})$, and thus there are no 'natural' canonical coordinates. The most widely used are Androyer-Deprit canonical coordinates [8] or the Euler angles and conjugated momenta. In fact we checked which, from almost all known coordinates on $T^{*} \mathrm{SO}(3, \mathbb{R})$, are most feasible for application of the Morales-Ramis theory. In our exposition we work with the Euler-Poisson equations. However, our choice of the body fixed frame is not conventional. Usually the principal axes frame is used. To see what is an advantage of our choice, let us notice that using the principal axes frame we can derive the normal variational equation in the form similar to (9), however, to put it in the form of an equation with rational coefficient we have to choose a transformation different than (10), and as a result, we obtain, instead of equation (12) possessing four regular singularities, a much more complicated Fuchsian equation with seven singular points. Our choice of the body fixed frame appears e.g. in [11].

Simplifications of the normal variational equations which occur when $L_{3}=0$ need

an explanation. In fact, one can observe that although the Riemann surface $\Gamma_{k}^{1}$ for the second particular solution is a torus with two points removed, see formulae (29), the normal variational equation (21) corresponding to it has the form of a Lamé equation, 
so it is defined over a torus with one point removed. The reason of what happened is symmetry. When $L_{3}=0$ the Euler-Poisson equations restricted to $M_{0}$ are invariant with respect to an involutive symplectic diffeomorphism $\mathcal{J}_{1}: M_{0} \mapsto M_{0}$ defined by

$$
\mathcal{J}_{1}\left(M_{1}, M_{2}, M_{3}, N_{1}, N_{2}, N_{3}\right)=\left(-M_{1}, M_{2},-M_{3}, N_{1},-N_{2}, N_{3}\right) \text {. }
$$

Let us denote

$$
M=\left\{p \in M_{0} \mid \mathcal{J}_{1}(p) \neq p\right\}, \quad \widehat{M}=M / \mathcal{J}_{1},
$$

and let $\pi: M \mapsto \widehat{M}$ be the projection. In the natural way equations (1) induce Hamiltonian equations on $\widehat{M}$ with Hamiltonian function $\widehat{H}=H \circ \pi^{-1}$. Then, according to Ziglin, see Lemma on page 36 in [50], if system (1) is integrable, then the induced Hamiltonian system on $\widehat{M}$ is also integrable. For the induced system we have a family of particular solutions $\widehat{\varphi}_{1}(t, k)=\pi \circ \varphi_{1}(t, k)$. The corresponding Riemann surfaces $\widehat{\Gamma}_{k}^{1}$ are tori with one point removed.

A simplification of the normal variational equation for solution $\varphi(t, k)$ when $L_{3}=0$ and the fact that we can transform them to a Riemann $P$ equation (for an appropriate choice of energy) is also related with symmetry. Namely, when $L_{3}=0$, system (1) restricted to $M_{0}$ is also invariant with respect to an involutive symplectic diffeomorphism $\mathcal{J}: M_{0} \mapsto M_{0}$ defined by

$$
\mathcal{J}\left(M_{1}, M_{2}, M_{3}, N_{1}, N_{2}, N_{3}\right)=\left(-M_{1},-M_{2}, M_{3}, N_{1}, N_{2},-N_{3}\right) .
$$

For symmetry reduction of variational equations see Section 4.2 in [4].

Let us note that in Lemma 2 we claim that if $d \neq 0$ then the identity component of the differential Galois group of equation (12) is not Abelian. Thus, it can be the whole group $\operatorname{SL}(2, \mathbb{C})$ or whole triangular subgroup $\mathcal{T}$ of $\operatorname{SL}(2, \mathbb{C})$. We do not know if the second case can occur.

In the case of first particular solution we do not work with the elliptic curve $\Gamma_{k}$ but with the Riemann sphere (minus singular point) for which $\Gamma_{k}$ is a covering. The reason of this is that we have no tool similar to the Kovacic algorithm for a second order linear differential equation defined on an elliptic curve. However, in the case of the second particular solution we can work directly on elliptic curve $\Gamma_{k}^{1}$ because, in this case, the normal variational equation is the Lamé equation for which the monodromy group is know. Of course, in this case we can also work on the Riemann sphere making well know transformation of the Lamé equation to its algebraic form.

In his proof of Theorem 2 and 3 Ziglin used the explicit time parametrisation of particular solutions. First he showed that if the system is integrable then the monodromy of the normal variational equations along real periods of a particular solution must be equal to the identity. Then, using analytical tools he derived the necessary conditions for the integrability.

In our exposition we use the explicit time parametrisation of particular solutions in the proof of Theorem 3. In fact, we use it only to show explicitly what happens with loops along real and imaginary periods when $k$ tends to 1 . However, one can deduce this information from the equations defining the elliptic curve. Thus, we can avoid using explicit time parametrisation at all. We keep it in the proof of Theorem 3 because, as we hope, it makes the exposition more transparent. 
The physical restriction $C \in(0,2)$ plays crucial role in our, as well as, in the Ziglin proof. Integrable systems are really rare, hence it is an interesting question if the EulerPoisson equations are integrable for values of parameters which do not satisfy this restriction.

Considering the case $L_{3}=0$ and the first particular solution Ziglin showed that the necessary condition for integrability is $c \in \mathbb{N}$. In our Proposition 1 there are two families of $c$ such that $c \in \mathbb{Q}$. The reason why they appears is that we fixed the energy for the first solution.

In the proof of Lemma 3 we show that the Brioschi-Halphen-Crawford case is possible only when $C \in\{1 / 4,3 / 4,5 / 4,7 / 4\}$. For this values of $C$ we can calculate the Brioschi determinant $Q_{m}\left(g_{2}, g_{3}, \beta\right)$ defined by (36). Calculations show that it vanishes identically only when $C=1 / 4$, i.e., for the Goryachev-Chaplygin case. Thus, in fact, to prove that for $C \in\{3 / 4,5 / 4,7 / 4\}$ the Euler-Poisson equations are non-integrable, we can use the second solution. We use the first one because calculations are simpler.

\section{Acknowledgements}

We are very thankful to Michèle Audin for her remarks, comments, suggestions and corrections. They allowed us to improve considerably not only the contents of the paper, but also gave a more clear proof of our main result.

We would like to thank Delphine Boucher, Juan J. Morales-Ruiz, Jacques-Arthur Weil, Carles Simó, Michael F. Singer and Felix Ulmer for discussions and help which allowed us to understand many topics related to this work. We are very grateful to Mark van Hoeij with whom we started to discuss some problems concerning this paper in the end of the previous century. Many important comments by Robert S. Maier concerning the Lamé equation are gratefully acknowledged.

As usual, we thank Zbroja (Urszula Maciejewska) not only for her linguistic help.

For the second author this research has been supported by a Marie Curie Fellowship of the European Community programme Human Potential under contract number HPMF-CT-2002-02031.

\section{Appendix}

\subsection{Dependence on a parameter}

Let us consider a second order differential equation of the following form

$$
y^{\prime \prime}=r(z, \varepsilon) y, \quad ' \equiv \frac{\mathrm{d}}{\mathrm{d} z},
$$

where $r(z, \varepsilon)$ is a rational function with respect to $z$ and $\varepsilon$, i.e., $r \in \mathbb{C}(\varepsilon)(z)=\mathbb{C}(z, \varepsilon)$. Here $\varepsilon$ plays the role of a parameter. For a fixed value of $\varepsilon$ we denote by $\mathcal{G}^{0}(\varepsilon)$ the identity component of the differential Galois group of equation (31). Let $U \subset \mathbb{C}$ denote an open not empty connected set with compact closure. We show the following.

Lemma 7. Assume that: 
1. Equation (31) is Fuchsian.

2. For $\varepsilon \in U$, equation (31) possesses $N$ singular points ( $N$ does not depend on $\varepsilon$ ) for which exponents do not depend on $\varepsilon$.

3. For $\varepsilon_{0} \in U, \mathcal{G}^{0}\left(\varepsilon_{0}\right)$ is not solvable (is not Abelian).

Then, except finitely many values of $\varepsilon \in U, \mathcal{G}^{0}(\varepsilon)$ is not solvable (is not Abelian).

Proof. From the Kovacic algorithm in the form given in [13; 38] we know that, under our assumption, if $\mathcal{G}^{0}(\varepsilon)$ is solvable, then there exists a polynomial $P$ (whose degree does not depend on $\varepsilon$ ) which is a solution of a linear differential equation $L(y)=0$ with coefficients in $\mathbb{C}(z, \varepsilon)$. The order of $L(y)=0$ does not depend on $\varepsilon$. We have a finite number of choices for the degree of $P$ and a finite number of choices of $L(y)=0$. Finding a polynomial solution of linear equation $L(y)=0$ reduces to finding a nontrivial solution of a homogeneous linear system with coefficients in $\mathbb{C}(\varepsilon)$. But the last problem reduces to finding common zeros of a finite number of polynomials. We know that not all of these polynomials vanish identically (otherwise $\mathcal{G}^{0}\left(\varepsilon_{0}\right)$ is solvable). Thus, there is at most a finite number of values of $\varepsilon$ for which they vanish simultaneously. Finally, let us notice that set

$$
\left\{\varepsilon \in U \mid \mathcal{G}^{0}(\varepsilon) \text { is Abelian }\right\},
$$

is a subset of

$$
\left\{\varepsilon \in U \mid \mathcal{G}^{0}(\varepsilon) \text { is solvable }\right\}
$$

\subsection{Second order differential equations with rational coefficients}

Let us consider a second order differential equation of the following form

$$
y^{\prime \prime}=r y, \quad r \in \mathbb{C}(z), \quad ' \equiv \frac{\mathrm{d}}{\mathrm{d} z} .
$$

For this equation its differential Galois group $\mathcal{G}$ is an algebraic subgroup of $\operatorname{SL}(2, \mathbb{C})$. The following lemma describes all possible types of $\mathcal{G}$ and relates these types to forms of solution of (32), see [19; 38].

Lemma 8. Let $\mathcal{G}$ be the differential Galois group of equation (32). Then one of four cases can occur.

1. $\mathcal{G}$ is reducible (it is conjugated to a subgroup of triangular group); in this case equation (32) has an exponential solution of the form $y=\exp \int \omega$, where $\omega \in \mathbb{C}(z)$,

2. $\mathcal{G}$ is conjugated with a subgroup of

$$
D^{\dagger}=\left\{\left[\begin{array}{cc}
c & 0 \\
0 & c^{-1}
\end{array}\right] \mid c \in \mathbb{C}^{*}\right\} \cup\left\{\left[\begin{array}{cc}
0 & c \\
c^{-1} & 0
\end{array}\right] \mid c \in \mathbb{C}^{*}\right\}
$$

in this case equation (32) has a solution of the form $y=\exp \int \omega$, where $\omega$ is algebraic over $\mathbb{C}(z)$ of degree 2 , 
3. $\mathcal{G}$ is primitive and finite; in this case all solutions of equation (32) are algebraic,

4. $\mathcal{G}=\operatorname{SL}(2, \mathbb{C})$ and equation (32) has no Liouvillian solution.

We need a more precise characterisation of case 1 in the above lemma. It is given by the following lemma, see Lemma 4.2 in [42].

Lemma 9. Let $\mathcal{G}$ be the differential Galois group of equation (32) and assume that $\mathcal{G}$ is reducible. Then either

1. equation (32) has a unique solution y such that $y^{\prime} / y \in \mathbb{C}(z)$, and $\mathcal{G}$ is conjugate to a subgroup of the triangular group

$$
\mathcal{T}=\left\{\left[\begin{array}{cc}
a & b \\
0 & a^{-1}
\end{array}\right] \mid a, b \in \mathbb{C}, a \neq 0\right\} .
$$

Moreover, $\mathcal{G}$ is a proper subgroup of $\mathcal{T}$ if and only if there exists $m \in \mathbb{N}$ such that $y^{m} \in \mathbb{C}(z)$. In this case $\mathcal{G}$ is conjugate to

$$
\mathcal{T}_{m}=\left\{\left[\begin{array}{cc}
a & b \\
0 & a^{-1}
\end{array}\right] \mid a, b \in \mathbb{C}, a^{m}=1\right\},
$$

where $m$ is the smallest positive integer such that $y^{m} \in \mathbb{C}(z)$, or

2. equation (32) has two linearly independent solutions $y_{1}$ and $y_{2}$ such that $y_{i}^{\prime} / y_{i} \in \mathbb{C}(z)$, then $\mathcal{G}$ is conjugate to a subgroup of

$$
\mathcal{D}=\left\{\left[\begin{array}{cc}
a & 0 \\
0 & a^{-1}
\end{array}\right] \mid a \in \mathbb{C}, a \neq 0\right\} .
$$

In this case, $y_{1} y_{2} \in \mathbb{C}(z)$. Furthermore, $\mathcal{G}$ is conjugate to a proper subgroup of $\mathcal{D}$ if and only if $y_{1}^{m} \in \mathbb{C}(z)$ for some $m \in \mathbb{N}$. In this case $\mathcal{G}$ is a cyclic group of order $m$ where $m$ is the smallest positive integer such that $y_{1}^{m} \in \mathbb{C}(z)$.

In case 2 of the above lemma we know that $v=y_{1} y_{2} \in \mathbb{C}(z)$. Differentiating $v$ three times, and using the fact that $y_{i}$ satisfies equation (32), we obtain

$$
v^{\prime \prime \prime}=2 r^{\prime} v+4 r v^{\prime}
$$

The above equation is called the second symmetric power of equation (32). For applications of symmetric powers of differential operators to study the existence of Liouvillian solutions and differential Galois group see e.g. [42; 43; 46].

To decide if case 2 from Lemma 8 occurs we can apply the Kovacic algorithm. Here we present its part devoted to this case and adopted to a Fuchsian equation. At first we introduce notation. We write $r(z) \in \mathbb{C}(z)$ in the form

$$
r(z)=\frac{s(z)}{t(z)}, \quad s(z), t(z) \in \mathbb{C}[z],
$$

where $s(z)$ and $t(z)$ are relatively prime polynomials and $t(z)$ is monic. The roots of $t(z)$ are poles of $r(z)$. We denote $\Sigma^{\prime}:=\{c \in \mathbb{C} \mid t(c)=0\}$ and $\Sigma:=\Sigma^{\prime} \cup\{\infty\}$. The 
order ord $(c)$ of $c \in \Sigma^{\prime}$ is equal to the multiplicity of $c$ as a root of $t(z)$, the order of infinity is defined by

$$
\operatorname{ord}(\infty):=\max (0,4+\operatorname{deg} s-\operatorname{deg} t) .
$$

Because we assume that equation (32) is Fuchsian, we have ord $(c) \leq 2$ for $c \in \Sigma$. For each $c \in \Sigma^{\prime}$ we have the following expansion

$$
r(z)=\frac{a_{c}}{(z-c)^{2}}+O\left(\frac{1}{z-c}\right),
$$

and we define $\Delta_{c}=\sqrt{1+4 a_{c}}$. For infinity we have

$$
r(z)=\frac{a_{\infty}}{z^{2}}+O\left(\frac{1}{z^{3}}\right),
$$

and we define $\Delta_{\infty}=\sqrt{1+4 a_{\infty}}$.

The algorithm consists of three steps.

Step I. For $c \in \Sigma^{\prime}$ such that $\operatorname{ord}(c)=1$ we define $E_{c}=\{4\}$; if $\operatorname{ord}(c)=2$

$$
E_{c}:=\left\{2,2\left(1+\Delta_{c}\right), 2\left(1-\Delta_{c}\right)\right\} \cap \mathbb{Z} .
$$

If $\operatorname{ord}(\infty)<2$ we put $E_{\infty}=\{0,2,4\}$; if $\operatorname{ord}(\infty)=2$ we define

$$
E_{\infty}:=\left\{2,2\left(1+\Delta_{\infty}\right), 2\left(1-\Delta_{\infty}\right)\right\} \cap \mathbb{Z} .
$$

Step II. For each $e$ in the Cartesian product

$$
E:=E_{\infty} \times \prod_{c \in \Sigma^{\prime}} E_{c}
$$

we compute

$$
d(e):=\frac{1}{2}\left(e_{\infty}-\sum_{c \in \Sigma^{\prime}} e_{c}\right) .
$$

We select those elements $e \in E$ for which $d(e)$ is a non-negative integer. If there are no such elements Case 2 from Lemma 8 cannot occur and the algorithm stops here.

Step III. For each element $e \in E$ such that $d(e)=n \in \mathbb{N}_{0}$ we define

$$
\theta=\theta(z)=\frac{1}{2} \sum_{c \in \Sigma^{\prime}} \frac{e_{c}}{z-c^{\prime}}
$$

and we search for a monic polynomial $P=P(z)$ of degree $n$ satisfying the following equation

$$
P^{\prime \prime \prime}+3 \theta P^{\prime \prime}+\left(3 \theta^{2}+3 \theta^{\prime}-4 r\right) P^{\prime}+\left(\theta^{\prime \prime}+3 \theta \theta^{\prime}+\theta^{3}-4 r \theta-2 r^{\prime}\right) P=0 .
$$

If such polynomial exists, then equation (32) possesses a solution of the form $w=$ $\exp \int \omega$, where

$$
\omega^{2}+\psi \omega+\frac{1}{2} \psi^{\prime}+\frac{1}{2} \psi^{2}-r=0, \quad \psi=\theta+\frac{P^{\prime}}{P} .
$$

If we do not find such polynomial, then case 2 in Lemma 8 cannot occur. 


\subsection{Riemann $P$ equation}

The Riemann $P$ equation [48] is the most general second order differential equation with three regular singularities. If we place, using homography, these singularities at $z=0,1, \infty$, then it has the form

$$
\begin{aligned}
\frac{\mathrm{d}^{2} \xi}{\mathrm{d} z^{2}} & +\left(\frac{1-\alpha-\alpha^{\prime}}{z}+\frac{1-\gamma-\gamma^{\prime}}{z-1}\right) \frac{\mathrm{d} \xi}{\mathrm{d} z} \\
& +\left(\frac{\alpha \alpha^{\prime}}{z^{2}}+\frac{\gamma \gamma^{\prime}}{(z-1)^{2}}+\frac{\beta \beta^{\prime}-\alpha \alpha^{\prime}-\gamma \gamma^{\prime}}{z(z-1)}\right) \xi=0,
\end{aligned}
$$

where $\left(\alpha, \alpha^{\prime}\right),\left(\gamma, \gamma^{\prime}\right)$ and $\left(\beta, \beta^{\prime}\right)$ are the exponents at singular points. Exponents satisfy the Fuchs relation

$$
\alpha+\alpha^{\prime}+\gamma+\gamma^{\prime}+\beta+\beta^{\prime}=1 .
$$

We denote differences of exponents by

$$
\lambda=\alpha-\alpha^{\prime}, \quad \gamma=\gamma-\gamma^{\prime}, \quad \mu=\beta-\beta^{\prime} .
$$

For equation (34) the necessary and sufficient conditions for solvability of the identity component of its differential Galois group are given by the following theorem due to Kimura [17], see also [38].

Theorem 5 (Kimura). The identity component of the differential Galois group of equation (34) is solvable if and only if

A: at least one of four numbers $\lambda+\mu+\nu,-\lambda+\mu+\nu, \lambda-\mu+\nu, \lambda+\mu-v$ is an odd integer, or

$B$ : the numbers $\lambda$ or $-\lambda$ and $\mu$ or $-\mu$ and $v$ or $-v$ belong (in an arbitrary order) to some of the following fifteen families

\begin{tabular}{|c|c|c|c|l|}
\hline 1 & $1 / 2+l$ & $1 / 2+s$ & arbitrary complex number & \\
\hline 2 & $1 / 2+l$ & $1 / 3+s$ & $1 / 3+q$ & \\
\hline 3 & $2 / 3+l$ & $1 / 3+s$ & $1 / 3+q$ & $l+s+q$ even \\
\hline 4 & $1 / 2+l$ & $1 / 3+s$ & $1 / 4+q$ & \\
\hline 5 & $2 / 3+l$ & $1 / 4+s$ & $1 / 4+q$ & $l+s+q$ even \\
\hline 6 & $1 / 2+l$ & $1 / 3+s$ & $1 / 5+q$ & \\
\hline 7 & $2 / 5+l$ & $1 / 3+s$ & $1 / 3+q$ & $l+s+q$ even \\
\hline 8 & $2 / 3+l$ & $1 / 5+s$ & $1 / 5+q$ & $l+s+q$ even \\
\hline 9 & $1 / 2+l$ & $2 / 5+s$ & $1 / 5+q$ & $l+s+q$ even \\
\hline 10 & $3 / 5+l$ & $1 / 3+s$ & $1 / 5+q$ & $l+s+q$ even \\
\hline 11 & $2 / 5+l$ & $2 / 5+s$ & $2 / 5+q$ & $l+s+q$ even \\
\hline 12 & $2 / 3+l$ & $1 / 3+s$ & $1 / 5+q$ & $l+s+q$ even \\
\hline 13 & $4 / 5+l$ & $1 / 5+s$ & $1 / 5+q$ & $l+s+q$ even \\
\hline 14 & $1 / 2+l$ & $2 / 5+s$ & $1 / 3+q$ & $l+s+q$ even \\
\hline 15 & $3 / 5+l$ & $2 / 5+s$ & $1 / 3+q$ & $l+s+q$ even \\
\hline
\end{tabular}

Here $l, s$ and $q$ are integers.

The solvability conditions are sufficient for our purposes because if $\mathcal{G}^{0}$ is not solvable, then obviously it is not Abelian. 


\subsection{Lamé equation}

The Weierstrass form of the Lamé equation is following

$$
\frac{\mathrm{d}^{2} y}{\mathrm{~d} t^{2}}=\left(\alpha \wp\left(t ; g_{2}, g_{3}\right)+\beta\right) y
$$

where $\alpha$ and $\beta$ are, in general, complex parameters and $\wp\left(t ; g_{2}, g_{3}\right)$ is the elliptic Weierstrass function with invariants $g_{2}, g_{3}$. In other words, $\wp\left(t ; g_{2}, g_{3}\right)$ is a solution of the differential equation

$$
\dot{v}^{2}=f(v), \quad f(v)=4 v^{3}-g_{2} v-g_{3} .
$$

It is assumed that equation $f(v)=0$ has three different roots, so

$$
\Delta=g_{2}^{3}-27 g_{3}^{2} \neq 0 .
$$

We recall that the modular function $j\left(g_{2}, g_{3}\right)$ associated with the elliptic curve $u^{2}=$ $4 v^{3}-g_{2} v-g_{3}$ is defined as follows

$$
j\left(g_{2}, g_{3}\right)=\frac{g_{2}^{2}}{g_{2}^{3}-27 g_{3}^{2}} .
$$

Classically the Lamé equation is written with parameter $n$ instead of $\alpha$ related by the formula $\alpha=n(n+1)$. We see that the Lamé equation depends on four parameters $\left(n, \beta, g_{2}, g_{3}\right)$. The following lemma lists all the cases in which the identity component of the differential Galois group of Lamé equation (35) is Abelian, see [38, Sec. 2.8.4].

Lemma 10. The identity component of the differential Galois group of Lamé equation (35) is Abelian only in the following cases:

1. the Lamé-Hermite case when $n \in \mathbb{Z}$ and three other parameters are arbitrary.

2. the Brioschi-Halphen-Crowford case for which $m:=n+\frac{1}{2} \in \mathbb{N}$, and remaining parameters $\left(g_{2}, g_{3}, \beta\right)$ satisfy an algebraic equation

$$
Q_{m}\left(g_{2}, g_{3}, \beta\right)=0,
$$

see below.

3. the Baldassarri case $2 n \notin \mathbb{Z}$, and

$$
n \pm q \in \mathbb{Z} \quad \text { for some } q \in\{1 / 4,1 / 6,1 / 10,3 / 10\}
$$

with additional algebraic restrictions on $\left(g_{2}, g_{3}, \beta\right)$.

Polynomial $Q_{m}\left(g_{2}, g_{3}, \beta\right)$ which appears in the Brioschi-Halphen-Crowford case, called the Brioschi determinant, is defined as follows

$$
\left|\begin{array}{ccccccc}
\beta & m-1 & 0 & 0 & 0 & \ldots & 0 \\
q_{2,1} & \beta & 2(m-2) & 0 & 0 & \ldots & 0 \\
q_{3,1} & q_{3,2} & \beta & 3(m-3) & 0 & \ldots & 0 \\
0 & q_{4,2} & q_{4,3} & \beta & 4(m-4) & \ldots & 0 \\
0 & 0 & \ddots & \ddots & \ddots & \ldots & 0 \\
0 & 0 & \ldots & q_{m-2, m-3} & q_{m-1, m-2} & \beta & m-1 \\
0 & 0 & \ldots & 0 & q_{m, m-2} & q_{m, m-1} & \beta
\end{array}\right|,
$$


where

$$
q_{i+1, i}=\frac{g_{2}}{4}(2 m-i)(m-i), \quad q_{i+2, i}=\frac{g_{3}}{4}(2 m-i)(2 m-i-1) .
$$

Algebraic restrictions on $\left(g_{2}, g_{3}, \beta\right)$ in the Baldassarri case are involved. Instead of them we use the following proposition which follows from one unpublished result of B. Dwork, see [38].

Proposition 2. The Baldassarri case for equation (35) occurs only for a finite number of values of $j\left(g_{2}, g_{3}\right)$.

\section{References}

[1] Arnold, V. I., Mathematical Methods of Classical Mechanics, Graduate Texts in Mathematics, Springer-Verlag, New York, 1978.

[2] Audin, M., Spinning tops, volume 51 of Cambridge Studies in Advanced Mathematics, Cambridge University Press, Cambridge, 1996.

[3] Baider, A. and Churchill, R. C., On monodromy groups of second-order Fuchsian equations, SIAM J. Math. Anal., 21(6):1642-1652, 1990.

[4] Baider, A., Churchill, R. C., Rod, D. L., and Singer, M. F., On the infinitesimal geometry of integrable systems, in Mechanics Day (Waterloo, ON, 1992), volume 7 of Fields Inst. Commun., pages 5-56, Amer. Math. Soc., Providence, RI, 1996.

[5] Beukers, F., Differential Galois theory, in From Number Theory to Physics (Les Houches, 1989), pages 413-439, Springer, Berlin, 1992.

[6] Bolotin, S. V., Double asymptotic trajectories and conditions for integrability of Hamiltonian systems, Vestnik Moskov. Univ. Ser. I Mat. Mekh., (1):55-63, 1990.

[7] Bolotin, S. V., Variational methods for constructing chaotic motions in the dynamics of a rigid body, Prikl. Mat. Mekh., 56(2):230-239, 1992.

[8] Borisov, A. and Mamaev, I., Dynamics of rigid body., NITS Regulyarnaya i Khaoticheskaya Dinamika, Izhevsk, 2001, in Russian.

[9] Churchill, R. C. and Rod, D. L., On the determination of Ziglin monodromy groups, SIAM J. Math. Anal., 22(6):1790-1802, 1991.

[10] Churchill, R. C., Rod, D. L., and Singer, M. F., Group-theoretic obstructions to integrability, Ergodic Theory Dynam. Systems, 15(1):15-48, 1995.

[11] Dokshevich, A. I., Resheniya v konechnom vide uravnenii Eilera-Puassona, "Naukova Dumka", Kiev, 1992.

[12] Dovbysh, S. A., Splitting of separatrices of unstable uniform rotations and nonintegrability of a perturbed Lagrange problem, Vestnik Moskov. Univ. Ser. I Mat. Mekh., (3):70-77, 1990. 
[13] Duval, A. and Loday-Richaud, M., Kovacic's algorithm and its application to some families of special functions, Appl. Algebra Engrg. Comm. Comput., 3(3):211246, 1992.

[14] Golubev, V. V., Lectures on Integration of Equations of Motion of a Rigid Body about a Fixed Point, Gosud. Isdat. Teh. Teor. Lit., Moscow, 1953, in Russian.

[15] Goryachev, D. I., New integrable cases of integrability of the Euler dynamical equations, Warsaw Univ. Izv., 1910, in Russian.

[16] Kaplansky, I., An Introduction to Differential Algebra, Hermann, Paris, second edition, 1976.

[17] Kimura, T., On Riemann's equations which are solvable by quadratures, Funkcial. Ekvac., 12:269-281, 1969/1970.

[18] Kirillov, A. A., Elements of the theory of representations, Springer-Verlag, Berlin, 1976, translated from the Russian by Edwin Hewitt, Grundlehren der Mathematischen Wissenschaften, Band 220.

[19] Kovacic, J. J., An algorithm for solving second order linear homogeneous differential equations, J. Symbolic Comput., 2(1):3-43, 1986.

[20] Kowalevski, S., Sur le problème de la rotation d'un corps solide autour d'un point fixe, Acta Math., 12:177-232, 1888.

[21] Kowalevski, S., Sur une propriété du systéme d'équations différentielles qui définit la rotation d'un corps solide autour d'un point fixe, Acta Math., 14:81-93, 1890.

[22] Kozlov, V. V., The nonexistence of an additional analytic integral in the problem of the motion of a nonsymmetric heavy solid body around a fixed point, Vestnik Moskov. Univ. Ser. I Mat. Mekh., (1):105-110, 1975.

[23] Kozlov, V. V., Methods of Qualitative Analysis in the Dynamics of a Rigid Body, Moskov. Gos. Univ., Moscow, 1980.

[24] Kozlov, V. V. and Treshchev, D. V., Nonintegrability of the general problem of rotation of a dynamically symmetric heavy rigid body with a fixed point. I, Vestnik Moskov. Univ. Ser. I Mat. Mekh., (6):73-81, 1985.

[25] Kozlov, V. V. and Treshchev, D. V., Nonintegrability of the general problem of rotation of a dynamically symmetric heavy rigid body with a fixed point. II, Vestnik Moskov. Univ. Ser. I Mat. Mekh., (1):39-44, 1986.

[26] Lagrange, J. L., Mécanique analytique, volume 2, Chez La Veuve Desaint, Libraire, Paris, 1788.

[27] Leimanis, E., The General Problem of the Motion of Coupled Rigid Bodies about a Fixed Point, Springer-Verlag, Berlin, 1965. 
[28] Maciejewski, A. J., Non-integrability in gravitational and cosmological models. Introduction to Ziglin theory and its differential Galois extension, in A. J. Maciejewski and B. Steves, editors, The Restless Universe. Applications of Gravitational N-Body Dynamics to Planetary, Stellar and Galactic Systems, pages 361-385, 2001.

[29] Maciejewski, A. J., Non-integrability of a certain problem of rotational motion of a rigid satellite, in H. Prȩtka-Ziomek, E. Wnuk, P. K. Seidelmann, and D. Richardson, editors, Dynamics of Natural and Artificial Celestial Bodies, pages 187-192, Kluwer Academic Publisher, 2001.

[30] Maciejewski, A. J., Non-integrability of certain Hamiltonian systems. Applications of Morales-Ramis differential Galois extension of Ziglin theory, in T. Crespo and Z. Hajto, editors, Differential Galois Theory, volume 58, pages 139-150, Banach Center Publication, Warsaw, 2002.

[31] Maciejewski, A. J. and Przybylska, M., Non-integrability of ABC flow, Phys. Lett. A, 303:265-272, 2002.

[32] Maciejewski, A. J. and Przybylska, M., Non-integrability of the Suslov problem, Regul. Chaotic Dyn., 7(1):73-80, 2002.

[33] Maciejewski, A. J. and Przybylska, M., Non-integrability of restricted two-body problems in constant curvature spaces, Regul. Chaotic Dyn., 8(4):413-430, 2003.

[34] Maciejewski, A. J. and Przybylska, M., Non-integrability of the problem of a rigid satellite in gravitational and magnetic fields, Celestial Mech., 87(4):317-351, 2003.

[35] Magid, A. R., Lectures on Differential Galois Theory, volume 7 of University Lecture Series, American Mathematical Society, Providence, RI, 1994.

[36] Marsden, J. E. and Ratiu, T. S., Introduction to Mechanics and Symmetry, number 17 in Texts in Applied Mathematics, Springer Verlag, New York, Berlin, Heidelberg, 1994.

[37] Morales, J. J. and Simó, C., Picard-Vessiot theory and Ziglin's theorem, J. Differential Equations, 107(1):140-162, 1994.

[38] Morales Ruiz, J. J., Differential Galois Theory and Non-Integrability of Hamiltonian Systems, volume 179 of Progress in Mathematics, Birkhäuser Verlag, Basel, 1999.

[39] Morales-Ruiz, J. J. and Ramis, J. P., Galoisian obstructions to integrability of Hamiltonian systems. I, Methods Appl. Anal., 8(1):33-95, 2001.

[40] Morales-Ruiz, J. J. and Ramis, J. P., Galoisian obstructions to integrability of Hamiltonian systems. II, Methods Appl. Anal., 8(1):97-111, 2001.

[41] Ramis, J.-P. and Martinet, J., Théorie de Galois différentielle et resommation, in Computer Algebra and Differential Equations, pages 117-214, Academic Press, London, 1990.

[42] Singer, M. F. and Ulmer, F., Galois groups of second and third order linear differential equations, J. Symbolic Comput., 16(1):9-36, 1993. 
[43] Singer, M. F. and Ulmer, F., Necessary conditions for Liouvillian solutions of (third order) linear differential equations, Appl. Algebra Engrg. Comm. Comput., 6(1):1-22, 1995.

[44] Souriau, J.-M., Structure des systèmes dynamiques, Maîtrises de mathématiques, Dunod, Paris, 1970.

[45] Takano, K. and Bannai, E., A global study of Jordan-Pochhammer differential equations, Funkcial. Ekvac., 19(1):85-99, 1976.

[46] Ulmer, F. and Weil, J.-A., Note on Kovacic's algorithm, J. Symbolic Comput., 22(2):179-200, 1996.

[47] van der Put, M. and Singer, M. F., Galois Theory of Linear Differential Equations, volume 328 of Grundlehren der Mathematischen Wissenschaften [Fundamental Principles of Mathematical Sciences], Springer-Verlag, Berlin, 2003.

[48] Whittaker, E. T. and Watson, G. N., A Course of Modern Analysis, Cambridge University Press, London, 1935.

[49] Ziglin, S. L., Branching of solutions and the nonexistence of an additional first integral in the problem of an asymmetric heavy rigid body in motion relative to a fixed point., Dokl. Akad. Nauk SSSR, 251(4):786-790, 1980.

[50] Ziglin, S. L., Branching of solutions and non-existence of first integrals in Hamiltonian mechanics. I, Functional Anal. Appl., 16:181-189, 1982.

[51] Ziglin, S. L., Branching of solutions and non-existence of first integrals in Hamiltonian mechanics. II, Functional Anal. Appl., 17:6-17, 1983.

[52] Ziglin, S. L., On the absence of a real-analytic first integral in some problems of dynamics, Functional Anal. Appl., 31(1):3-9, 1997. 